\title{
Spectral Computations on Lamplighter Groups and Diestel-Leader Graphs
}

\author{
Laurent Bartholdi and Wolfgang Woess \\ Communicated by Fulvio Ricci
}

\begin{abstract}
The Diestel-Leader graph DL $(q, r)$ is the horocyclic product of the homogeneous trees with respective degrees $q+1$ and $r+1$. When $q=r$, it is the Cayley graph of the lamplighter group (wreath product) $\mathbb{Z}_{q} 2 \mathbb{Z}$ with respect to a natural generating set. For the "Simple random walk" (SRW) operator on the latter group, Grigorchuk and $\dot{Z} u k$, and Dicks and Schick have determined the spectrum and the (on-diagonal) spectral measure (Plancherel measure). Here, we show that thanks to the geometric realization, these results can be obtained for all DL-graphs by directly computing an $\ell^{2}$-complete orthonormal system of finitely supported eigenfunctions of the SRW. This allows computation of all matrix elements of the spectral resolution, including the Plancherel measure. As one application, we determine the sharp asymptotic behavior of the $N$-step return probabilities of $S R W$. The spectral computations involve a natural approximating sequence of finite subgraphs, and we study the question whether the cumulative spectral distributions of the latter converge weakly to the Plancherel measure. To this end, we provide a general result regarding Folner approximations; in the specific case of $D L(q, r)$, the answer is positive only when $r=q$.
\end{abstract}

\section{Introduction}

Let $X$ be a locally finite connected graph. Simple random walk (SRW) on $X$ is the Markov chain on $X$ with transition probabilities

$$
p(x, y)= \begin{cases}1 / \operatorname{deg}(x) & \text { if } y \sim x, \\ 0 & \text { otherwise }\end{cases}
$$

Here, $\sim$ denotes neighborhood, and $\operatorname{deg}(x)$ is the degree (number of neighbors) of vertex $x \in X$. The transition operator associated with SRW acts on real or complex functions $f$

\footnotetext{
Math Subject Classifications. 05C50, 20E22, 47A10, 60B15.

Keywords and Phrases. Lamplighter group, wreath product, Diestel-Leader graph, random walk, spectrum, spectral measures.

Acknowledgements and Notes. Supported by FWF (Austrian Science Fund) project P15577.
} 
on $X$ by

$$
P f(x)=\sum_{y} p(x, y) f(y) .
$$

In particular, $P$ acts as a self-adjoint operator on the weighted $\ell^{2}$-space $\ell^{2}(X, \operatorname{deg})$ and has norm $=$ spectral radius $\leq 1$. Here, we shall only consider regular graphs, i.e., deg is constant, and we prefer to use the ordinary space $\ell^{2}(X)$, where the reference measure is the counting measure (instead of $m(x)=\operatorname{deg}(x)$ ).

Associated with $P$ there is the resolution of the identity. This is an operator-valued measure $E$ defined on $\operatorname{spec}(P)$ such that for all $N \geq 0$,

$$
P^{N}=\int_{\operatorname{spec}(P)} \lambda^{N} E(d \lambda) .
$$

It is characterized by the matrix elements

$$
\mu_{x, y}(d \lambda)=\left\langle\delta_{x}, E(d \lambda) \delta_{y}\right\rangle,
$$

which in turn are characterized by their moments, which are the $N$-step transition probabilities (matrix elements of $P^{N}$ ),

$$
p^{(N)}(x, y)=\int_{\operatorname{spec}(P)} \lambda^{N} \mu_{x, y}(d \lambda) \quad \forall N \geq 0 .
$$

Each $\mu_{x, x}$ is a probability measure, while the off-diagonal $\mu_{x, y}$ are signed measures with total mass 0 . When $X$ is a transitive graph (i.e., its group of isometries acts transitively on the vertex set), then all $\mu_{x, x}$ coincide, and we shall just write $\mu$ for this measure, whose support is the whole spectrum. This holds, in particular, for Cayley graphs of finitely generated groups. ${ }^{1}$ In the spirit of Harmonic Analysis, we call $\mu$ the Plancherel measure; more recently, it has also been called the Kesten spectral measure by some authors.

Basic references for the general theory of spectra of infinite graphs and groups are the articles of Mohar and Woess [20], de la Harpe, Robertson and Valette [12], and Grigorchuk and Żuk [13]. In [20] and [12], one can also find many examples of specific graphs and groups where spectra and (less frequently) spectral measures are computed: In basic cases $\operatorname{spec}(P)$ is an interval, and the Plancherel measure has a continuous density with respect to Lebesgue measure. This occurs for integer lattices - a classical result from Fourier analysis, see e.g., Pólya [22] — and for free groups, resp. homogeneous trees — see Kesten [15] and Cartier [5]. For other tree-like cases (infinite distance-regular graphs), the situation is almost the same, with a possible additional isolated eigenvalue, as was shown by Kuhn and Soardi [17]; see also Faraut and Picardello [11]. For $\tilde{A}_{d}$-buildings with arbitrary $d$, the situation is similar to that of homogeneous trees, see Cartwright and Młotkowski [7] and Cartwright [6].

The situation is different on typical fractal graphs such as the one associated with the Sierpiński gasket, where the spectrum is pure point, i.e., the closure of the set of eigenvalues of $P$, see Malozemov and Teplyaev [18], Teplyaev [27], Sabot [24] and, for a generalization, Krön [16]. These graphs are regular, but far from being transitive. However,

\footnotetext{
$\overline{{ }^{1} \text { If } \Gamma \text { is a group and } S}=S^{-1} \subset \Gamma$ a finite set of generators, then the Cayley graph $X(\Gamma, S)$ has vertex set $\Gamma$, and $x \sim y$, if $x^{-1} y \in S$.
} 
it turned out in recent research that a similar situation may also occur in certain classes of fractal groups related with the construction of Grigorchuk and Gupta and Sidki of finitely generated infinite torsion groups with intermediate growth. For a comprehensive survey of these groups and their properties, see Bartholdi, Grigorchuk and Nekrashevych [2] and the references given there, and for the specific computation of a pure point spectrum on such a group, see Bartholdi and Grigorchuk [1].

Coming finally to the types of structures considered in the present article, a pure point spectrum with the associated Plancherel (Kesten) measure was recently detected for a different class of groups, namely the lamplighter groups (wreath products) $F \geq \mathbb{Z}$, where $F$ is a finite group; see Grigorchuk and $\dot{Z}$ uk [14] for $\mathbb{Z}_{2} 2 \mathbb{Z}$, and Dicks and Schick [8] for the general case. ${ }^{2}$ While [14] uses approximation of the considered Cayley graph by an increasing sequence of finite graphs, [8] applies von-Neumann-algebraic methods.

Note that the present study of random walks on $F \geq \mathbb{Z}$ depends only on the cardinality $|F|$, whence it suffices to consider $F=\mathbb{Z}_{q}$. Now, the Cayley graphs of $\mathbb{Z}_{q} 2 \mathbb{Z}$ considered in [14] and [8] turn out to have geometric realizations as specific examples $D L(q, q)$ in the family of Diestel-Leader graphs introduced by Diestel and Leader [9], i.e., the horocyclic products $D L(q, r)$ of two homogeneous trees with degree $q+1$ and $r+1$, respectively; see Woess [29] and Section 2 below.

In the present article, we exploit this geometric model to provide in Section 3 a completely explicit and elementary construction of an $\ell^{2}$-complete orthonormal system of finitely supported eigenfunctions of the SRW-operator on $D L(q, r)$. This comprises the lamplighter groups, but holds more generally for all Diestel-Leader graphs. When $q \neq r$, the graph $D L(q, r)$ is transitive, but not a Cayley graph, whence the group-specific methods of [8] do not apply here (they rely on identifying eigenfunctions as projections in the von Neumann algebra of a group acting on the graph; here that group is non-discrete and non-unimodular), nor can one use an approximating sequence of Schreier graphs as in [14].

We recover the spectral radius of SRW on $D L(q, r)$, computed previously by SaloffCoste and Woess [25],

$$
\rho(P)=\frac{2 \sqrt{q r}}{q+r} .
$$

The spectrum $\operatorname{spec}(P)=[-\rho(P), \rho(P)]$ is the closure of the set of all eigenvalues

$$
\left\{\lambda_{m, n}=\rho(P) \cos \frac{m}{n} \pi: n \geq 2,1 \leq m \leq n-1\right\},
$$

see Theorem 1. This theorem can be used to find an expression for each of the spectral measures, see Section 4. In particular, the Plancherel measure can be computed explicitly (Corollary 2).

We then use the latter in Section 5 to determine the sharp asymptotic behavior of the $2 N$-step return probabilities $p^{(2 N)}(x, x)$, as $N \rightarrow \infty$, see Theorem 2 (note that $p^{(2 N+1)}(x, x)=0$ since $D L(q, r)$ is bipartite). For the lamplighter groups, i.e., on $\operatorname{DL}(q, q)$, these asymptotics have been determined for almost the same random walk by Revelle [23]; one has

$$
p^{(2 N)}(x, x) \sim \bar{A}_{1} \exp \left(-B_{1} N^{1 / 3}\right) N^{1 / 6} \quad(r=q) .
$$

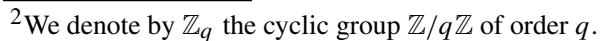


It is interesting to note that in the case $r \neq q$, the last (polynomial) term changes by a factor of $N$ :

$$
p^{(2 N)}(x, x) \sim A_{1} \rho(P)^{2 N} \exp \left(-B_{1} N^{1 / 3}\right) N^{-5 / 6} \quad(r \neq q) .
$$

Furthermore, the constants $\bar{A}_{1}, A_{1}, B_{1}>0$ are determined explicitly as functions of $q$ and $r$.

Next, in Section 6, we discuss for general vertex transitive graphs under which conditions the cumulative spectral measures of an approximating sequence of finite graphs converge (resp. do not converge) weakly to the Plancherel measure. A positive criterion is given in terms of $F \phi$ lner sequences of approximating subgraphs, and more generally, Følner approximations - see Theorem 3 and Remark 1. In our setting, this applies to $D L(q, q)$ with the natural subgraphs (tetrahedra) used in Section 3 for computing the spectrum, since they constitute a Følner sequence. However, when $r \neq q$, this is not true, and the cumulative spectral measures associated with tetrahedra do not converge to the Plancherel measure - see Proposition 3, which is preceded by lengthy computations of the spectra of tetrahedra. This should be seen in the light of amenability. A graph is called amenable, if it has a Følner sequence of subgraphs, which is equivalent with $\rho(P)=1$ for SRW. By [25], $D L(q, r)$ is amenable if and only if $r=q$; see (1.3) above. The discussion of Section 6 should be compared with the results of Serre [26], who studies (among other) the question under which conditions the cumulative spectral measures of an arbitrary sequence of regular graphs have a weak limit. (Our tetrahedra are not regular at their boundaries.)

At the end, in Section 7, we add several observations, including random walks with drift, the corresponding spectra, their return probabilities, and also their projections on the two subtrees and on $\mathbb{Z}$.

\section{Lamplighter Groups and Diestel-Leader Graphs}

This section is a short version of Section 2 in [29]. We explain the structure of the DL-graphs and their relation with the groups $\mathbb{Z}_{q} 2 \mathbb{Z}$.

Let $\mathbb{T}=\mathbb{T}_{q}$ be the homogeneous tree with degree $q+1, q \geq 2$. A geodesic path, respectively geodesic ray, respectively infinite geodesic in $\mathbb{T}$ is a finite, respectively one-sided infinite, respectively doubly infinite sequence $\left(x_{n}\right)$ of vertices of $\mathbb{T}$ such that $d\left(x_{i}, x_{j}\right)=|i-j|$ for all $i, j$, where $d(\cdot, \cdot)$ denotes the graph distance.

Two rays are equivalent, if the symmetric difference of their supports is finite. An end of $\mathbb{T}$ is an equivalence class of rays. The space of ends is denoted $\partial \mathbb{T}$, and we write $\widehat{\mathbb{T}}=\mathbb{T} \cup \partial \mathbb{T}$. For all $w, z \in \widehat{\mathbb{T}}$ there is a unique geodesic $\overline{w z}$ that connects the two. In particular, if $x \in \mathbb{T}$ and $\xi \in \partial \mathbb{T}$ then $\overline{x \xi}$ is the ray that starts at $x$ and represents $\xi$.

For $x, y \in \mathbb{T}, x \neq y$, we define the cone $\widehat{\mathbb{T}}(x, y)=\{w \in \widehat{\mathbb{T}}: y \in \overline{x w}\}$. The collection of all cones is the basis of a topology which makes $\widehat{\mathbb{T}}$ a compact, totally disconnected Hausdorff space with $\mathbb{T}$ as a dense, discrete subset.

We fix a root $o \in \mathbb{T}$. If $w, z \in \widehat{\mathbb{T}}$, then their confluent $c=w \wedge z$ with respect to the root vertex $o$ is defined by $\overline{o w} \cap \overline{o z}=\overline{o c}$. Similarly, we choose and fix a reference end $\omega \in \partial \mathbb{T}$. For $z, v \in \widehat{\mathbb{T}} \backslash\{\omega\}$, their confluent $b=v \curlywedge z$ with respect to $\omega$ is defined by $\overline{v \omega} \cap \overline{z \omega}=\overline{b \omega}$. We write

$$
z \preccurlyeq v \quad \text { if } \quad z \curlywedge v=z .
$$

For $x \in \mathbb{T}$, we describe its relative position with respect to $o$ by the two numbers

$$
\mathfrak{u}(x)=d(o, x \curlywedge o) \quad \text { and } \quad \mathfrak{d}(x)=d(x, x \curlywedge o) .
$$


In Figure $1, \mathfrak{u}(x)$ and $\mathfrak{d}(x)$ correspond to the numbers of steps one has to take upwards (in direction of $\omega$ ), respectively downwards, on the geodesic path from $o$ to $x$. Thus, $d(x, o)=\mathfrak{u}(x)+\mathfrak{d}(x)$.

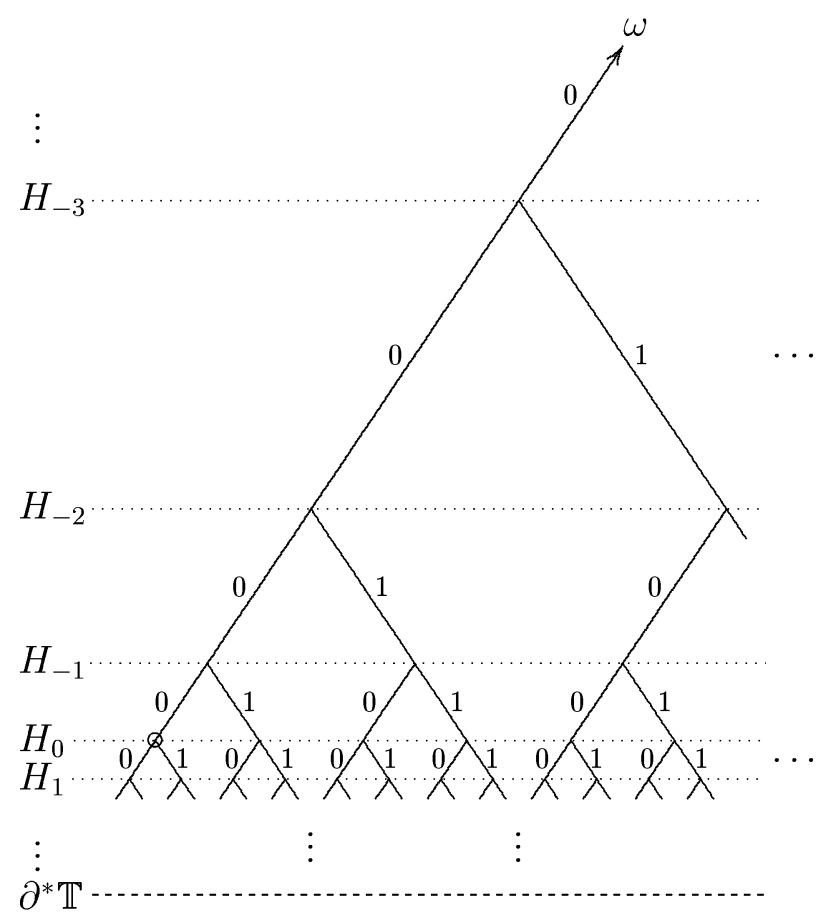

FIGURE 1

The Busemann function $\mathfrak{h}: \mathbb{T} \rightarrow \mathbb{Z}$ and the horocycles $H_{k}$ with respect to $\omega$ are defined as

$$
\mathfrak{h}(x)=\mathfrak{d}(x)-\mathfrak{u}(x) \quad \text { and } \quad H_{k}=\{x \in \mathbb{T}: \mathfrak{h}(x)=k\} .
$$

Every horocycle is infinite. Every vertex $x$ in $H_{k}$ has one neighbor $x^{-}$(its predecessor) in $H_{k-1}$ and $q$ neighbors (its successors) in $H_{k+1}$. We set $\partial^{*} \mathbb{T}=\partial \mathbb{T} \backslash\{\omega\}$.

We label each edge of $\mathbb{T}$ by an element of $\mathbb{Z}_{q}$ such that for each vertex, the "downward" edges to its $q$ successors carry labels $0, \ldots, q-1$ from left to right (say), see Figure 1 . Thus, for each $x \in \mathbb{T}$, the sequence $(\sigma(n))_{n \leq 0}$ of labels on the geodesic $\overline{x \omega}$ has finite support $\{n: \sigma(n) \neq 0\}$. We write $\Sigma_{q}$ for the set of all those sequences. On every horocycle, there is exactly one vertex corresponding to each $\sigma \in \Sigma_{q}$. Thus, $\mathbb{T}_{q}$ is in one-to-one correspondence with the set $\Sigma_{q} \times \mathbb{Z}$, and the $k$-th horocycle is $H_{k}=\Sigma_{q} \times\{k\}$.

Now consider two trees $\mathbb{T}^{1}=\mathbb{T}_{q}$ and $\mathbb{T}^{2}=\mathbb{T}_{r}$ with roots $o_{1}$ and $o_{2}$ and reference ends $\omega_{1}$ and $\omega_{2}$, respectively.

Definition 1. The Diestel-Leader graph $D L(q, r)$ is

$$
D L(q, r)=\left\{x_{1} x_{2} \in \mathbb{T}_{q} \times \mathbb{T}_{r}: \mathfrak{h}\left(x_{1}\right)+\mathfrak{h}\left(x_{2}\right)=0\right\},
$$

and neighborhood is given by $x_{1} x_{2} \sim y_{1} y_{2} \Longleftrightarrow x_{1} \sim y_{1}$ and $x_{2} \sim y_{2}$. 
To visualize $D L(q, r)$, draw $\mathbb{T}_{q}$ in horocyclic layers with $\omega_{1}$ at the top and $\partial^{*} \mathbb{T}_{q}$ at the bottom, and right to it $\mathbb{T}_{r}$ in the same way, but upside down, with the respective horocycles $H_{k}\left(\mathbb{T}_{q}\right)$ and $H_{-k}\left(\mathbb{T}_{r}\right)$ aligned. Connect the two origins $o_{1}, o_{2}$ by an elastic spring. It is allowed to move along each of the two trees, may expand infinitely, but must always remain in horizontal position. The vertex set of $D L_{q, r}$ consists of all admissible positions of the spring. From a position $x_{1} x_{2}$ with $\mathfrak{h}\left(x_{1}\right)+\mathfrak{h}\left(x_{2}\right)=0$ the spring may move downwards to one of the $r$ successors of $x_{2}$ in $\mathbb{T}_{r}$, and at the same time to the predecessor of $x_{1}$ in $\mathbb{T}_{q}$, or it may move upwards in the analogous way. Such a move corresponds to going to a neighbor of $x_{1} x_{2}$. We see that $D L(q, r)$ is regular with degree $q+r$. As the reference point in $D L(q, r)$, we choose $o=o_{1} o_{2}$. Figure 2 illustrates $D L(2,2)$.

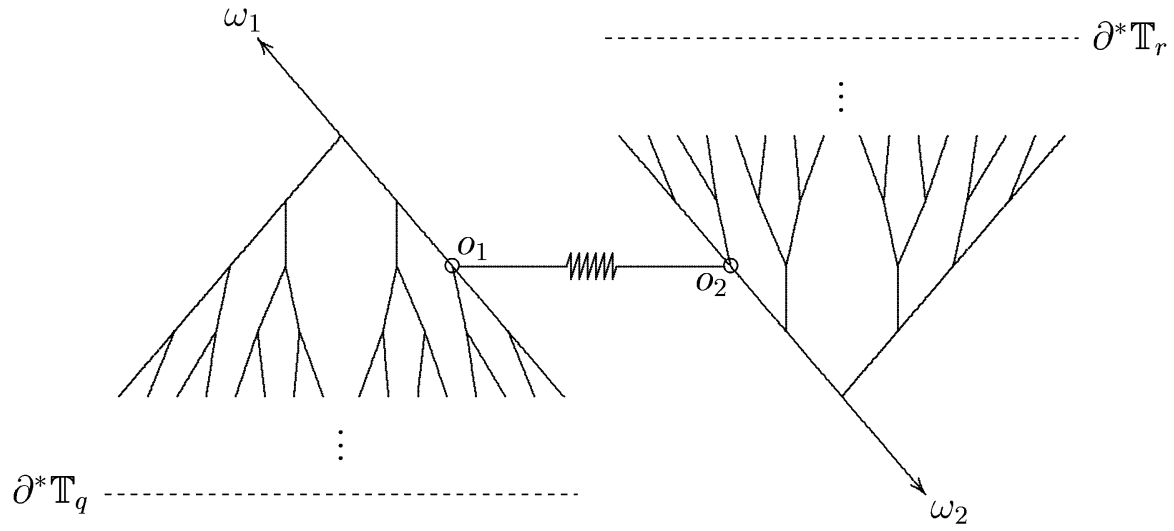

FIGURE 2

The relative position of $x=x_{1} x_{2} \in D L(q, r)$ with respect to $o$ is given by the four numbers $\mathfrak{u}\left(x_{1}\right), \mathfrak{d}\left(x_{1}\right), \mathfrak{u}\left(x_{2}\right), \mathfrak{d}\left(x_{2}\right)$, which satisfy the relation

$$
\mathfrak{u}\left(x_{1}\right)+\mathfrak{u}\left(x_{2}\right)=\mathfrak{d}\left(x_{1}\right)+\mathfrak{d}\left(x_{2}\right) .
$$

The lamplighter group $\mathbb{Z}_{q} 2 \mathbb{Z}$ is defined as follows: Consider the group of all finitely supported configurations

$$
\mathcal{C}=\left\{\eta: \mathbb{Z} \rightarrow \mathbb{Z}_{q}, \quad|\operatorname{supp}(\eta)|<\infty\right\}
$$

with pointwise addition modulo $q$. Then the group $\mathbb{Z}$ acts on $\mathcal{C}$ by translations $k \mapsto T_{k}$ : $\mathcal{C} \rightarrow \mathcal{C}$ with $T_{k} \eta(m)=\eta(m-k)$. The resulting semidirect product $\mathbb{Z} \wedge \mathcal{C}$ is

$\mathbb{Z}_{q} 2 \mathbb{Z}=\{(\eta, k): \eta \in \mathcal{C}, k \in \mathbb{Z}\} \quad$ with group operation $(\eta, k)\left(\eta^{\prime}, k^{\prime}\right)=\left(\eta+T_{k} \eta^{\prime}, k+k^{\prime}\right)$.

We identify each $(\eta, k) \in \mathbb{Z}_{q} 2 \mathbb{Z}$ with the vertex $x_{1} x_{2} \in D L(q, q)$, where according to the identification $\mathbb{T}_{q} \leftrightarrow \Sigma_{q} \times \mathbb{Z}$, the vertices $x_{i}$ are given by

$$
\begin{aligned}
x_{1} & =\left(\eta_{k}^{-}, k\right) \quad \text { and } \quad x_{2}=\left(\eta_{k}^{+},-k\right), \quad \text { where } \\
\eta_{k}^{-} & =\left.\eta\right|_{(-\infty, k]} \quad \text { and } \quad \eta_{k}^{+}=\left.\eta\right|_{[k+1, \infty)},
\end{aligned}
$$

both written as sequences over the non-positive integers.

This is clearly a one-to-one correspondence between $\mathbb{Z}_{q} 2 \mathbb{Z}$ and $D L(q, q)$, and it is also straightforward that this group acts transitively and fixed-point-freely on the graph. The 
action of $m \in \mathbb{Z}$ is given by $x_{1} x_{2}=\left(\sigma_{1}, k\right)\left(\sigma_{2},-k\right) \mapsto y_{1} y_{2}=\left(\sigma_{1}, k+m\right)\left(\sigma_{2},-k-m\right)$, and the action of the group of configurations is pointwise addition modulo $q$. Write $\delta_{k}^{\ell}$ for the configuration in $\mathcal{C}$ with value $\ell$ at $k$ and 0 elsewhere. Then $D L(q, q)$ is the (right) Cayley graph of $\mathbb{Z}_{q} 2 \mathbb{Z}$ with respect to the symmetric set of generators

$$
\left\{\left(\delta_{1}^{\ell}, 1\right),\left(\delta_{0}^{\ell},-1\right): \ell \in \mathbb{Z}_{q}\right\}
$$

i.e., an edge corresponds to multiplying with a generator on the right. This is precisely the set of generators considered in [14] and [8] when computing the spectrum of the associated SRW-operator.

\section{Tetrahedra and Horizontal Functions}

In the sequel, we shall often write $D L$ for $D L(q, r)$. We say that a function $f: D L \rightarrow \mathbb{C}$ is horizontal if it is finitely supported and

$$
\sum_{y_{2} \in \mathbb{T}^{2}: \mathfrak{h}\left(y_{2}\right)=-\mathfrak{h}\left(x_{1}\right)} f\left(x_{1} y_{2}\right)=\sum_{y_{1} \in \mathbb{T}^{1}: \mathfrak{h}\left(y_{1}\right)=-\mathfrak{h}\left(x_{2}\right)} f\left(y_{1} x_{2}\right)=0 \quad \forall x_{1} \in \mathbb{T}^{1}, x_{2} \in \mathbb{T}^{2}
$$

Lemma 1. The linear space of horizontal functions is dense in $\ell^{2}(D L)$.

Proof. It is sufficient to show that every point mass can be approximated in the $\ell^{2}$-norm by horizontal functions. Furthermore, by vertex-transitivity, it is sufficient to show this for $\delta_{o}$ where $o=o_{1} o_{2}$. Let $b_{1}=b_{1}^{n} \in \mathbb{T}^{1}$ be a vertex on $H_{-n}^{1}$ (horocycle in $\mathbb{T}^{1}$ ) for which $\mathfrak{u}\left(b_{1}\right)=n+1$ and $\mathfrak{d}\left(b_{1}\right)=1$. Define a function $f_{1}=f_{1}^{n}$ on $\mathbb{T}^{1}$ by

$$
f_{1}\left(x_{1}\right)= \begin{cases}1 & \text { if } \quad x_{1}=o_{1}, \\ -1 / q^{n} & \text { if } \quad b_{1} \preccurlyeq x_{1} \in H_{0}^{1}, \\ 0 & \text { otherwise }\end{cases}
$$

In the same way, but replacing $q$ with $r$, we define a function $f_{2}=f_{2}^{n}$ on $\mathbb{T}^{2}$. Then the function $f=f_{n}$, given by $f\left(x_{1} x_{2}\right)=f_{1}\left(x_{1}\right) f_{2}\left(x_{2}\right)$, is horizontal, and

$$
\left\|f_{n}-\delta_{o}\right\|^{2}=\frac{1}{q^{n} r^{n}}+\frac{1}{q^{n}}+\frac{1}{r^{n}} \rightarrow 0 \quad \text { as } \quad n \rightarrow \infty .
$$

Definition 2. Let $a_{1} \in \mathbb{T}^{1}$ and $a_{2} \in \mathbb{T}^{2}$ be two vertices with $-\mathfrak{h}\left(a_{2}\right)=\mathfrak{h}\left(a_{1}\right)+n$, where $n \geq 0$. Then the (induced) subgraph of $D L$ given by

$$
S=S\left(a_{1}, a_{2}\right)=\left\{x_{1} x_{2} \in D L: a_{1} \preccurlyeq x_{1}, a_{2} \preccurlyeq x_{2}\right\}
$$

is called a tetrahedron in $D L$ with height $n(S)=n$.

We shall only be interested in computations on tetrahedra with height $n \geq 2$.

If for $i=1,2$ we write $S^{i}=S_{n}^{i}\left(a_{i}\right)=\left\{x_{i} \in \mathbb{T}^{i}: a_{i} \preccurlyeq x_{i}, d\left(x_{i}, a_{i}\right) \leq n\right\}$, then $S=\left\{x_{1} x_{2} \in S_{1} \times S_{2}: \mathfrak{h}\left(x_{1}\right)+\mathfrak{h}\left(x_{2}\right)=0\right\}$. The boundary of $S^{i}$ in $\mathbb{T}^{i}$ is $\left\{a_{i}\right\} \cup \partial^{*} S^{i}$, where $\partial^{*} S^{1}=\left\{b_{1} \in S^{1}: b_{1} a_{2} \in S\right\}$, respectively $\partial^{*} S^{2}=\left\{b_{2} \in S^{2}: a_{1} b_{2} \in S\right\}$, and the boundary of $S$ in $D L$ is

$$
\partial S=\left(\left\{a_{1}\right\} \times \partial^{*} S^{2}\right) \cup\left(\partial^{*} S^{1} \times\left\{a_{2}\right\}\right) .
$$




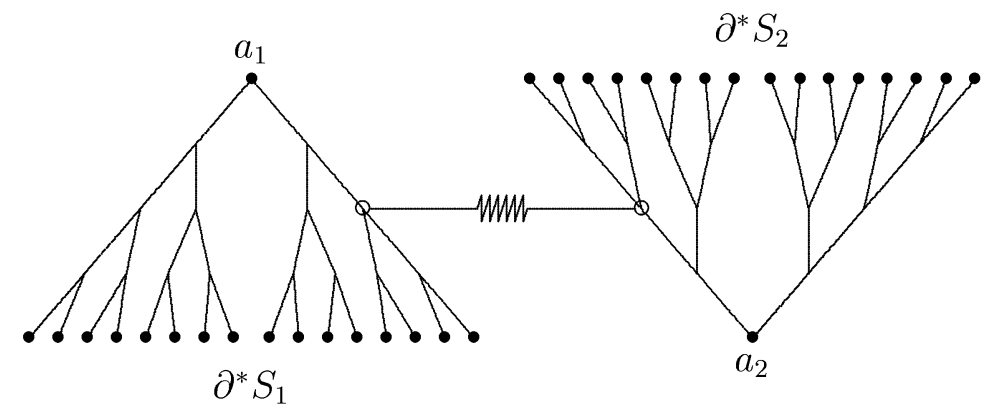

\section{FIGURE 3}

Imagining $S$ as a tetrahedron, two of its faces are copies of $S^{1}$ that meet at the common bottom side $\partial^{*} S_{1} \times\left\{a_{2}\right\}$, and the other two faces are copies of $S^{2}$ that meet at the common top side $\left\{a_{1}\right\} \times \partial^{*} S_{2}$. For $k=0, \ldots, n$, the $k$-th level of $S=S\left(a_{1}, a_{2}\right)$ is the set

$L_{k}=L_{k}\left(a_{1}, a_{2}\right)=\left\{x_{1} x_{2} \in S: \mathfrak{h}\left(x_{1}\right)=\mathfrak{h}\left(a_{1}\right)+k\right\}=\left(S^{1} \cap H_{\mathfrak{h}\left(a_{1}\right)+k}^{1}\right) \times\left(S^{2} \cap H_{\mathfrak{h}\left(a_{2}\right)+n-k}^{2}\right)$.

It has $q^{k} r^{n-k}$ elements. Furthermore, we write $v_{1, s}$ for the successor of $a_{1}$ where the edge $\left[a_{1}, v_{1, s}\right]$ of $\mathbb{T}^{1}$ has label $s \in \mathbb{Z}_{q}$, and analogously $v_{2, t}$ for the successor of $a_{2}$ where the edge $\left[a_{2}, v_{2, t}\right]$ of $\mathbb{T}^{2}$ has label $t \in \mathbb{Z}_{r}$.

We identify functions on $S$ with their extensions to $D L$, where the latter have value 0 on $D L \backslash S$. In particular, every horizontal function on $S$ must be 0 on $\partial S$. We now choose non-zero functions $\varphi^{1}$ on $\mathbb{Z}_{q}$ and $\varphi^{2}$ on $\mathbb{Z}_{r}$ such that

$$
\sum_{s=0}^{q-1} \varphi^{1}(s)=\sum_{t=0}^{r-1} \varphi^{2}(t)=0 \quad \text { and } \quad \sum_{s=0}^{q-1}\left(\varphi^{1}(s)\right)^{2}=\sum_{t=0}^{r-1}\left(\varphi^{2}(t)\right)^{2}=1 .
$$

(Later on, we shall make specific choices for $\varphi^{1}$ and $\varphi^{2}$.) Using these two functions, we define functions $f_{k}^{1}=f_{k}^{1}\left[\varphi^{1}\right]$ on $\mathbb{T}^{1}\left(a_{1}\right)=\left\{x_{1} \in \mathbb{T}^{1}: a_{1} \preccurlyeq x_{1}\right\}$ and $f_{k}^{2}=f_{k}^{2}\left[\varphi^{2}\right]$ on $\mathbb{T}^{2}\left(a_{2}\right)=\left\{x_{2} \in \mathbb{T}^{1}: a_{2} \preccurlyeq x_{2}\right\}$ by $f_{0}^{1} \equiv 0$, respectively $f_{0}^{2} \equiv 0$, and for $k \geq 1$,

$$
\begin{aligned}
f_{k}^{1}\left(x_{1}\right) & =\left\{\begin{array}{ll}
\varphi^{1}(s) q^{(1-k) / 2} & \text { if } v_{1, s} \preccurlyeq x_{1} \in H_{\mathfrak{h}\left(a_{1}\right)+k}^{1}, \\
0 & \text { otherwise },
\end{array}\right. \text { and } \\
f_{k}^{2}\left(x_{2}\right) & = \begin{cases}\varphi^{2}(t) r^{(1-k) / 2} & \text { if } v_{2, t} \preccurlyeq x_{2} \in H_{\mathfrak{h}\left(a_{2}\right)+k}^{2}, \\
0 & \text { otherwise } .\end{cases}
\end{aligned}
$$

For $k \geq 1$, these functions have $\ell^{2}$-norm 1 . Now we define for $0 \leq k \leq n$

$$
f_{k, n}(x)=f_{k}\left[S, \varphi^{1}, \varphi^{2}\right](x)=f_{k}^{1}\left(x_{1}\right) f_{n-k}^{2}\left(x_{2}\right), \quad x=x_{1} x_{2} \in S=S\left(a_{1}, a_{2}\right) .
$$

Recall that $n=-\mathfrak{h}\left(a_{2}\right)-\mathfrak{h}\left(a_{1}\right) \geq 2$ is the height of $S$. The following is a straightforward exercise.

Lemma 2. The functions $f_{k, n}, k=1, \ldots, n-1$, are horizontal and orthonormal in $\ell^{2}(S)$. The $S R W$-operator P satisfies

$$
P f_{k, n}=\frac{\sqrt{q r}}{q+r}\left(f_{k-1, n}+f_{k+1, n}\right) .
$$


Thus, since $f_{k, 0}=f_{k, n}=0$, the action of $P$ on the linear space spanned by $f_{k, n}$, $k=1, \ldots, n-1$, is described by the $(n-1) \times(n-1)$ tridiagonal matrix

$$
M_{n-1}=\frac{\sqrt{q r}}{q+r}\left(\begin{array}{cccc}
0 & 1 & & \\
1 & \ddots & \ddots & \\
& \ddots & \ddots & 1 \\
& & 1 & 0
\end{array}\right)
$$

Its eigenvalues $\lambda_{m, n}$ and associated orthonormal eigenvectors $\psi_{m, n}$ (the latter written as functions on $\{1, \ldots, n-1\})$ are

$$
\begin{aligned}
\lambda_{m, n} & =\frac{2 \sqrt{q r}}{q+r} \cos \frac{m}{n} \pi \quad \text { and } \\
\psi_{m, n}(k) & =\sqrt{\frac{2}{n}} \sin \frac{k m}{n} \pi, \quad m, k=1, \ldots, n-1 .
\end{aligned}
$$

Corollary 1. The functions $g_{m, n}$ on $S, m=1, \ldots, n-1$, defined by

$$
g_{m, n}=\sum_{k=1}^{n-1} \psi_{m, n}(k) f_{k, n}
$$

are horizontal and orthonormal in $\ell^{2}(S)$ as well as in $\ell^{2}(D L)$. They satisfy

$$
\begin{aligned}
P g_{m, n} & =\lambda_{m, n} \cdot g_{m, n} \quad \text { and } \\
\operatorname{span}\left\{g_{m, n}: m=1, \ldots, n-1\right\} & =\operatorname{span}\left\{f_{k, n}: k=1, \ldots, n-1\right\} .
\end{aligned}
$$

Once more, recall that besides depending on the height $n$ of $S$, each $g_{m, n}$ depends on $a_{1}, a_{2}, \varphi^{1}$ and $\varphi^{2}$,

$$
g_{m, n}=g_{m}\left[S, \varphi^{1}, \varphi^{2}\right] .
$$

Lemma 3. Let $S\left(a_{1}, a_{2}\right)$ and $S\left(\tilde{a}_{1}, \tilde{a}_{2}\right)$ be two tetrahedra of heights $n$ and $\tilde{n} \geq 2$, respectively. Let $\varphi^{1}$ and $\varphi^{2}$, respectively $\tilde{\varphi}^{1}$ and $\tilde{\varphi}^{2}$ be as in (3.2). Write $f_{k, n}=f_{k}\left[a_{1}, a_{2}, \varphi^{1}, \varphi^{2}\right]$ and $\tilde{f}_{l, \tilde{n}}=f_{l}\left[\tilde{a}_{1}, \tilde{a}_{2}, \tilde{\varphi}^{1}, \tilde{\varphi}^{2}\right]$. If one of

(i) $\left(a_{1}, a_{2}\right) \neq\left(\tilde{a}_{1}, \tilde{a}_{2}\right)$, or

(ii) $\left(a_{1}, a_{2}\right)=\left(\tilde{a}_{1}, \tilde{a}_{2}\right)$ and $\varphi^{1} \perp \tilde{\varphi}^{1}$ or $\varphi^{2} \perp \tilde{\varphi}^{2}$

holds, then

$$
\operatorname{span}\left\{f_{k, n}: k=1, \ldots, n-1\right\} \perp \operatorname{span}\left\{\tilde{f}_{l, \tilde{n}}: l=1, \ldots, \tilde{n}-1\right\} \text {. }
$$

Proof. (i) If $S\left(a_{1}, a_{2}\right) \cap S\left(\tilde{a}_{1}, \tilde{a}_{2}\right)=\emptyset$ then perpendicularity is obvious.

If $S\left(a_{1}, a_{2}\right) \cap S\left(\tilde{a}_{1}, \tilde{a}_{2}\right) \neq \varnothing$ then both $a_{1}, \tilde{a}_{1}$ and $a_{2}, \tilde{a}_{2}$ must be comparable with respect to the partial order $\preccurlyeq$. Assume that $a_{1} \preccurlyeq \tilde{a}_{1}$ and $a_{1} \neq \tilde{a}_{1}$. (The other three cases are treated analogously). Let $\kappa=\mathfrak{h}\left(\tilde{a}_{1}\right)-\mathfrak{h}\left(a_{1}\right)$.

If $k \neq \kappa+l$ then we certainly have $f_{k, n} \perp \tilde{f}_{l, \tilde{n}}$ (since the two functions have disjoint support).

If $k=\kappa+l$ then by construction, the function $f_{k}^{1}$ on $\mathbb{T}^{1}\left(a_{1}\right)$, as defined in (3.3), is constant on supp $\tilde{f}_{l}^{1}$, where the latter is given as in (3.3), but on $\mathbb{T}^{1}\left(\tilde{a}_{1}\right)$. Since $\sum \tilde{f}_{l}^{1}=$ 
0 , we have $f_{k}^{1} \perp \tilde{f}^{1} l$, Therefore, with $f_{k}^{2}$ and $\tilde{f}_{l}^{2}$ defined in the same way on $\mathbb{T}^{2}\left(a_{2}\right)$, respectively $\mathbb{T}^{2}\left(\tilde{a}_{2}\right)$,

$$
\sum_{x} f_{k, n}(x) \tilde{f}_{l, \tilde{n}}(x)=\sum_{x_{2}} f_{n-k}^{2}\left(x_{2}\right) \tilde{f}_{\tilde{n}-l}^{2}\left(x_{2}\right) \underbrace{\sum_{x_{1}} f_{k}^{1}\left(x_{1}\right) \tilde{f}_{l}^{2}\left(x_{1}\right)}_{=0}=0 .
$$

(ii) If $\left(a_{1}, a_{2}\right)=\left(\tilde{a}_{1}, \tilde{a}_{2}\right)$ then $\tilde{n}=n$, and $f_{k, n} \perp \tilde{f}_{l, n}$, if $l \neq k$, since the functions have disjoint support. Finally,

$$
\left\langle f_{k, n}, \tilde{f}_{k, n}\right\rangle=\left\langle\varphi^{1}, \tilde{\varphi}^{1}\right\rangle\left\langle\varphi^{2}, \tilde{\varphi}^{2}\right\rangle=0 .
$$

We now specify our choices for the functions $\varphi^{1}$ and $\varphi^{2}$. For $i=1, \ldots, q-1$ and $s \in \mathbb{Z}_{q}$, let

$$
\varphi_{i}^{1}(s)= \begin{cases}\frac{q-i}{\sqrt{(q-i)(q+1-i)}}, & s=i-1, \\ -\frac{1}{\sqrt{(q-i)(q+1-i)}}, & s=i, \ldots, q-1, \\ 0, & \text { otherwise } .\end{cases}
$$

These functions are orthogonal and satisfy (3.2). Analogously, replacing $i$ with $j$ and $q$ with $r$, we define the orthogonal functions $\varphi_{j}^{2}(t), j=1, \ldots, r-1\left(t \in \mathbb{Z}_{r}\right)$. We shall write

$$
f_{k}^{[S, i, j]}=f_{k}\left[S, \varphi_{i}^{1}, \varphi_{j}^{2}\right] \quad \text { and } g_{m}^{[S, i, j]}=g_{m}\left[S, \varphi_{i}^{1}, \varphi_{j}^{2}\right] .
$$

Proposition 1. The set

$\mathfrak{B}_{S}=\left\{g_{m}^{[\tilde{S}, i, j]}: \tilde{S} \subseteq S ; m \in\{1, \ldots, n(\tilde{S})-1\} ; i \in\{1, \ldots, q-1\} ; j \in\{1, \ldots, r-1\}\right\}$ constitutes an orthonormal basis of the linear space of all horizontal functions on the tetrahedron $S$. Here, $\tilde{S}$ runs through all tetrahedra in $D L(q, r)$ that are contained in $S$ and have height $n(\tilde{S}) \geq 2$.

Proof. Instead of the functions $g_{m}^{[\tilde{S}, i, j]}$, we may equivalently work with the functions $f_{m}^{[\tilde{S}, i, j]}$, since they are also linearly independent and span the same space as $\mathfrak{B}_{S}$.

If $L_{k}$ is the $k$-th level of $S$ (where $S$ is assumed to have height $n$ ) and $f$ is any horizontal function with support in $L_{k}$, then $f$ must satisfy each of the following $q^{k}+r^{n-k}$ equations

$$
\sum_{x_{2}: x_{1} x_{2} \in L_{k}} f\left(x_{1} x_{2}\right)=0 \text { and } \sum_{x_{1}: x_{1} x_{2} \in L_{k}} f\left(x_{1} x_{2}\right)=0 .
$$

Thus, the dimension of the linear space of all horizontal functions with support in $L_{k}$ is $\left(q^{k}-1\right)\left(r^{n-k}-1\right)$.

On the other hand, we can count all $f_{m}^{[\tilde{S}, i, j]}$ where $\tilde{S} \subseteq S$ and supp $f_{m}^{[\tilde{S}, i, j]} \subseteq L_{k}$. We find $(q-1)(r-1)$ functions of this type (one for each pair $(i, j)$ ) associated with every tetrahedron $\tilde{S}=S\left(\tilde{a}_{1}, \tilde{a}_{2}\right)$, where $a_{1} \preccurlyeq \tilde{a}_{1}$ and $d\left(\tilde{a}_{1}, a_{1}\right) \leq k-1$, and at the same time $a_{2} \preccurlyeq \tilde{a}_{2}$ and $d\left(\tilde{a}_{2}, a_{2}\right) \leq n-k-1$. There are precisely

$$
\sum_{\kappa=0}^{k-1} q^{\kappa} \sum_{\nu=0}^{n-k-1} r^{\nu}=\frac{\left(q^{k}-1\right)\left(r^{n-k}-1\right)}{(q-1)(r-1)}
$$


choices for $\left(\tilde{a}_{1}, \tilde{a}_{2}\right)$. Thus, the number of all functions $f_{m}^{[\tilde{S}, i, j]}$ with support in $L_{k}$ (which are linearly independent) coincides with the dimension of the space of all horizontal functions on $L_{k}$, and we have a basis of that space. Putting together the different levels of $S$, we obtain the proposed result.

Thus, we obtain the following spectral decomposition of $\ell^{2}(D L)$.

Theorem 1. The spectrum of the SRW-operator $P$ on $D L(q, r)$ is given by the interval $[-\rho(P), \rho(P)]$, where $\rho(P)=2 \sqrt{q r} /(q+r)$.

It is a pure point spectrum, being the closure of the set

$$
\left\{\lambda_{m, n}=\rho(P) \cos \frac{m}{n} \pi: n \geq 2,1 \leq m \leq n-1\right\} .
$$

Furthermore, the set of all functions

$$
\mathfrak{B}=\left\{g_{m}^{[S, i, j]}: \begin{array}{c}
\text { S tetrahedron in } D L ; m \in\{1, \ldots, n(\tilde{S})-1\} ; \\
i \in\{1, \ldots, q-1\} ; j \in\{1, \ldots, r-1\}
\end{array}\right\},
$$

constructed in Corollary 1, respectively (3.8), is a complete orthonormal system in $\ell^{2}(D L)$ consisting of finitely supported functions; we have

$$
P g_{m}^{[S, i, j]}=\lambda_{m, n} \cdot g_{m}^{[S, i, j]},
$$

where $n=n(S)$ is the height of $S$.

Proof. If $f$ is any horizontal function on $D L$, then there is some tetrahedron $S$ containing its support. By Proposition $1, f$ is a linear combination of elements of $\mathfrak{B}_{S}$. Thus, $\mathfrak{B}=$ $\bigcup_{S} \mathfrak{B}_{S}$ is an orthonormal system that spans the space of all horizontal functions. Now Lemma 1 completes the proof.

\section{The Spectral Measures}

Using Theorem 1, we can compute the spectral measures (1.1). Indeed, if $x \in D L(q, r)$, then the Fourier expansion of $\delta_{x}$ with respect to the orthonormal system of Theorem 1 is

$$
\delta_{x}=\sum_{S} \sum_{m=1}^{n(S)-1} \sum_{i=1}^{q-1} \sum_{j=1}^{r-1} g_{m}^{[S, i, j]}(x) g_{m}^{[S, i, j]}(\cdot) .
$$

Therefore, for $x, y \in D L$,

$$
p^{(N)}(x, y)=\left\langle\delta_{x}, P^{N} \delta_{y}\right\rangle=\sum_{S} \sum_{m=1}^{n(S)-1} \sum_{i=1}^{q-1} \sum_{j=1}^{r-1} \lambda_{m, n(S)}^{N} g_{m}^{[S, i, j]}(x) g_{m}^{[S, i, j]}(y),
$$

and comparing this with (1.2), we find that for any continuous function $\mathbf{f}$ on $\operatorname{spec}(P)$, its integral with respect to $\mu_{x, y}$ is

$$
\int_{\operatorname{spec}(P)} \mathbf{f}(\lambda) \mu_{x, y}(d \lambda)=\sum_{S} \sum_{m=1}^{n(S)-1} \sum_{i=1}^{q-1} \sum_{j=1}^{r-1} \mathbf{f}\left(\lambda_{m, n(S)}\right) g_{m}^{[S, i, j]}(x) g_{m}^{[S, i, j]}(y)
$$


a countable sum of point masses. Since $D L$ is transitive, we only need the measures $\mu_{o, x}$, where $x \in D L$. Let $x=x_{1} x_{2}$ with $\mathfrak{u}_{i}=\mathfrak{u}\left(x_{i}\right)$ and $\mathfrak{d}_{i}=\mathfrak{d}\left(x_{i}\right)$. Furthermore, for $i=1,2$ let $c_{i}=x_{i} \curlywedge o_{i}$, so that $S\left(c_{1}, c_{2}\right)$ has height $\mathfrak{s}=\mathfrak{u}_{1}+\mathfrak{u}_{2}=\mathfrak{d}_{1}+\mathfrak{d}_{2}=\left(d\left(x_{1}, o_{1}\right)+d\left(x_{2}, o_{2}\right)\right) / 2$, see Figure 4.

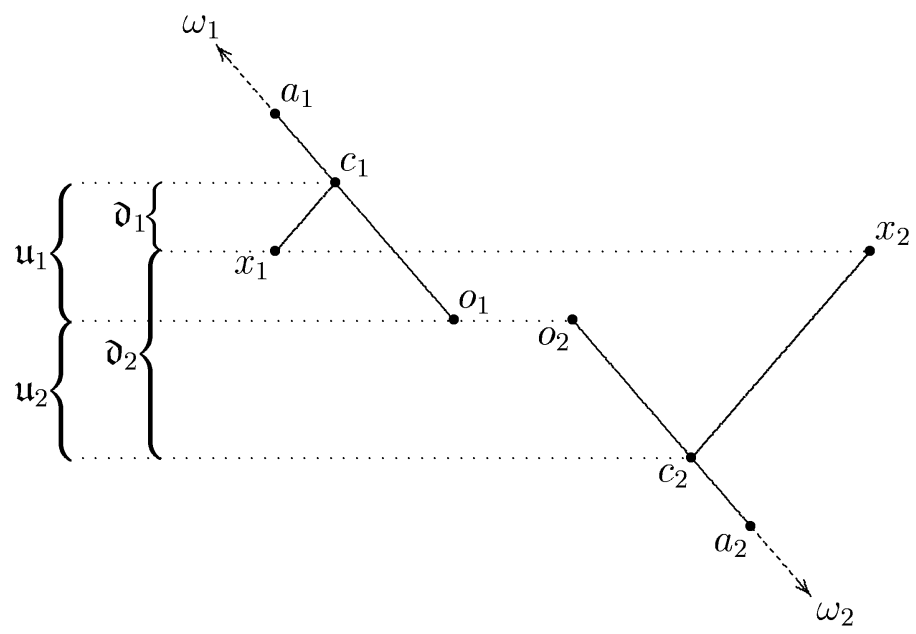

FIGURE 4

Since $\lambda_{m, n}$ depends only on $m / n$, we choose $m$ and $n$ relatively prime $(1 \leq m<n)$. In order to compute the mass $\mu_{o, x}\left(\lambda_{m, n}\right)$, we have to consider all tetrahedra $S=S\left(a_{1}, a_{2}\right)$ with height $n(S)=\ell n$ that contain $S\left(c_{1}, c_{2}\right)$, that is, $a_{i} \in \overline{c_{i} \omega_{i}}$ for $i=1,2$. For those $S$, note that $g_{m}^{[S, i, j]}(o)=0$ when $(i, j) \neq(1,1)$. Therefore, denoting by $\lceil\cdot\rceil$ the next larger integer, (4.1) now yields

$$
\mu_{o, x}\left(\lambda_{m, n}\right)=\sum_{\ell=\lceil\mathfrak{s} / n\rceil}^{\infty} \sum_{\substack{S \supseteq S\left(c_{1}, c_{2}\right) \\ n(S)=\ell n}} g_{\ell m}^{[S, 1,1]}(o) g_{\ell m}^{[S, 1,1]}(x),
$$

and, if we set

$$
k_{i}=k_{i}(S)=d\left(a_{i}, c_{i}\right)=\mathfrak{h}\left(c_{i}\right)-\mathfrak{h}\left(a_{i}\right), \quad i=1,2,
$$

then by Corollary 1

$$
\begin{aligned}
g_{\ell m}^{[S, 1,1]}(x) g_{\ell m}^{[S, 1,1]}(o)= & C_{k_{1}, k_{2}} q^{-k_{1}-\left(\mathfrak{u}_{1}+\mathfrak{d}_{1}\right) / 2} r^{-k_{2}-\left(\mathfrak{u}_{2}+\mathfrak{d}_{2}\right) / 2} \\
& \times \frac{2}{\ell n} \sin \left(\left(k_{1}+\mathfrak{u}_{1}\right) \frac{m}{n} \pi\right) \sin \left(\left(k_{1}+\mathfrak{d}_{1}\right) \frac{m}{n} \pi\right) .
\end{aligned}
$$

where $n(S)=\ell n$ and

$$
C_{k_{1}, k_{2}}= \begin{cases}(q-1)(r-1), & \text { if } k_{1}>0 \quad \text { and } k_{2}>0 \\ -(r-1), & \text { if } k_{1}=0 \text { and } k_{2}>0 \\ -(q-1), & \text { if } k_{1}>0 \text { and } k_{2}=0 \\ 1, & \text { if } k_{1}=k_{2}=0\end{cases}
$$


The last case occurs only when $a_{1}=c_{1}$ and $a_{2}=c_{2}$. Also, note that $k_{1}+k_{2}=n(S)-\mathfrak{s}$ and that $\mathfrak{u}_{i}+\mathfrak{d}_{i}=d\left(x_{i}, o_{i}\right), i=1,2$. We obtain

Proposition 2. If $1 \leq m<n$ and $m$ and $n$ are relatively prime, then with constants as in (4.4),

$$
\begin{aligned}
& \mu_{o, x}\left(\lambda_{m, n}\right) \\
& =\sum_{\ell=\lceil\mathfrak{s} / n\rceil}^{\infty} \frac{2 r^{-\ell n}}{\ell n} \sum_{k=0}^{\ell n-\mathfrak{s}} C_{k, \ell n-\mathfrak{s}-k}(r / q)^{k+d\left(x_{1}, o_{1}\right) / 2} \sin \left(\left(k+\mathfrak{u}_{1}\right) \frac{m}{n} \pi\right) \sin \left(\left(k+\mathfrak{d}_{1}\right) \frac{m}{n} \pi\right) .
\end{aligned}
$$

Elementary computations yield the following.

Corollary 2. The Plancherel measure $\mu=\mu_{o, o}$ is given by

$$
\mu\left(\lambda_{m, n}\right)= \begin{cases}\frac{\log \left(1-r^{-n}\right)-\log \left(1-q^{-n}\right)}{n(r-q)} \frac{2 q r(q+r)(q-1)(r-1) \sin ^{2} \frac{m}{n} \pi}{(r-q)^{2}+4 q r \sin ^{2} \frac{m}{n} \pi}, & \text { if } r \neq q, \\ \frac{(q-1)^{2}}{q^{n}-1}, & \text { if } r=q,\end{cases}
$$

where $\lambda_{m, n}=\frac{2 \sqrt{q r}}{q+r} \cos \frac{m}{n} \pi$ and $m$ and $n$ are relatively prime $(1 \leq m<n)$.

Note that $\lambda_{n-m, n}=-\lambda_{m, n}$ and $\mu\left(\lambda_{n-m, n}\right)=\mu\left(-\lambda_{m, n}\right)$, that is, the Plancherel measure is symmetric, as it has to be, since $D L(q, r)$ is a bipartite graph (it has no odd cycles). The formula for $\mu$ in the case $r=q$ (lamplighter group) was obtained previously in [14] and [8]. For $x \neq o$, the inner sum in Proposition 2 can be computed in (lengthy) closed form, but in general not the outer one.

\section{Asymptotic Behavior of the Return Probabilities}

Combining (1.2) with Corollary 2, we can determine the exact asymptotic behavior of the return probabilities $p^{(N)}(o, o)$ as $N \rightarrow \infty$. For odd $N$, these probabilities are $=0$.

For dealing with $p^{(2 N)}(o, o)$, the following standard technical lemma will be useful.

Lemma 4. For $k \in \mathbb{N}$ and $\gamma \in \mathbb{R}$ and any sequence $\varepsilon_{n}$ tending to 0 , let

$$
\Sigma(N)=\Sigma(N ; k, \gamma)=\sum_{n=2 k+1}^{\infty}\left(1+\varepsilon_{n}\right) n^{\gamma} q^{-n} \cos ^{2 N} \frac{k}{n} \pi .
$$

Then

$$
\Sigma(N) \sim \xi_{k}^{\gamma}\left(2 \pi / C_{k}\right)^{1 / 2} \exp \left(-B_{k} N^{1 / 3}\right) N^{(1+2 \gamma) / 6} \text { as } N \rightarrow \infty,
$$

where

$$
\begin{aligned}
& \Phi_{k}(\xi)=\xi \log q+\frac{(k \pi)^{2}}{\xi^{2}}, \quad \xi_{k}=\left(\frac{2(k \pi)^{2}}{\log q}\right)^{1 / 3} ; \\
& B_{k}=\Phi_{k}\left(\xi_{k}\right)=3\left(\frac{k \pi \log q}{2}\right)^{2 / 3}, \quad \Phi_{k}^{\prime}\left(\xi_{k}\right)=0 \quad \text { and } \quad C_{k}=\Phi_{k}^{\prime \prime}\left(\xi_{k}\right)=6\left(\frac{(\log q)^{2}}{4 k \pi}\right)^{2 / 3} .
\end{aligned}
$$

(Here, as usual, $\sim$ denotes asymptotic equivalence, i.e., quotients tending to 1 . It will always be clear from the context whether $\sim$ means neighborhood in a graph or asymptotic equivalence.) 
Proof. We decompose $\Sigma(N)=\Sigma_{1}(N)+\Sigma_{2}(N)$, where the sum $\Sigma_{1}(N)$ ranges over all $n$ with $2 k<n<\kappa N^{1 / 3}$, and $\Sigma_{2}(N)$ ranges over all $n \geq \kappa N^{1 / 3}$, and where $\kappa=\xi_{k} / 2$.

We start with $\Sigma_{2}(N)$. For $n \rightarrow \infty$,

$$
\cos ^{2 N} \frac{k}{n} \pi=\exp \left(-(k \pi)^{2} N\left(n^{-2}+O\left(n^{-4}\right)\right)\right) .
$$

Therefore, we have as $N \rightarrow \infty$

$$
\Sigma_{2}(N) \sim N^{\gamma / 3} \sum_{n \geq \kappa N^{1 / 3}} \xi_{N, n}^{\gamma} \exp \left(-N^{1 / 3} \Phi_{k}\left(\xi_{N, n}\right)\right), \quad \text { where } \quad \xi_{N, n}=\frac{n}{N^{1 / 3}} .
$$

The point where $\Phi_{k}$ attains its minimum is $\xi_{k}$, and we compute the values $\Phi_{k}\left(\xi_{k}\right)=B_{k}$ and $\Phi_{k}^{\prime \prime}\left(\xi_{k}\right)=C_{k}$, as given above. Therefore

$$
\Phi_{k}(\xi)=B_{k}+\frac{C_{k}}{2}\left(\xi-\xi_{k}\right)^{2}+R(\xi)\left(\xi-\xi_{k}\right)^{3}
$$

with $R(\xi)$ continuous for $\xi>0$. We now "substitute"

$$
\tau_{N, n}=N^{1 / 6}\left(\xi_{N, n}-\xi_{k}\right), \quad \text { with } \quad \Delta \tau_{N, n}=\tau_{N, n+1}-\tau_{N, n}=N^{-1 / 6} \rightarrow 0 .
$$

Then we can rewrite (5.1) as

$$
\begin{aligned}
\Sigma_{2}(N) \sim & \exp \left(-B_{k} N^{1 / 3}\right) N^{(1+2 \gamma) / 6} \\
& \times \sum_{\substack{n: \\
\tau_{N, n} \geq-\kappa N^{1 / 6}}}\left(\xi_{k}+N^{-1 / 6} \tau_{N, n}\right)^{\gamma} \exp \left\{-\frac{C}{2} \tau_{N, n}^{2}-R_{N}^{*}\left(\tau_{N, n}\right)\right\} \Delta \tau_{N, n}
\end{aligned}
$$

where $R_{N}^{*}(\tau)=N^{-1 / 6} R\left(\xi_{0}+N^{-1 / 6} \tau\right) \tau^{3}$.

It is now standard that the sum in (5.2) converges to $\xi_{k}^{\gamma} \int_{-\infty}^{\infty} e^{-C_{k} \tau^{2} / 2} d \tau=\xi_{k}^{\gamma}$ $\sqrt{2 \pi / C}$. (One has to use dominated convergence in a suitable central piece of the sum and control the two tails.) Thus, $\Sigma_{2}(N)$ has the asymptotic behavior that we have proposed for $\Sigma(N)$.

Let us now look at $\Sigma_{1}(N)$. Set $M=\sup _{n}\left|1+\varepsilon_{n}\right| n^{\gamma} q^{-n}$. Then

$$
\Sigma_{1}(N) \leq M \kappa N^{1 / 3} \cos ^{2 N}\left(\frac{k \pi}{\kappa N^{1 / 3}}\right) \sim M \kappa N^{1 / 3} \exp \left(-\frac{(k \pi)^{2}}{\kappa^{2}} N^{1 / 3}\right) .
$$

With our choice $\kappa=\xi_{k} / 2$, one checks that $(k \pi)^{2} / \kappa^{2}>B_{k}$. Therefore $\Sigma_{1}(N) / \Sigma_{2}(N) \rightarrow 0$ as $N \rightarrow \infty$.

For the following, recall from Theorem 1 that $\rho(P)=1$ when $r=q$. When $r \neq q$, it is enough to consider only $r>q$.

Theorem 2. Let $\xi_{1}, B_{1}$ and $C_{1}$ be as defined in Lemma 4.

(i) If $r>q$ then

$$
p^{(2 N)}(o, o) \sim A_{1} \rho(P)^{2 N} \exp \left(-B_{1} N^{1 / 3}\right) N^{-5 / 6} \quad \text { as } N \rightarrow \infty,
$$

where

$$
A_{1}=4 \pi^{2} \xi_{1}^{-3}\left(2 \pi / C_{1}\right)^{1 / 2} q r(q+r)(q-1)(r-1) /(r-q)^{3} .
$$


(ii) If $r=q$ then

$$
p^{(2 N)}(o, o) \sim \bar{A}_{1} \exp \left(-B_{1} N^{1 / 3}\right) N^{1 / 6} \quad \text { as } N \rightarrow \infty,
$$

where

$$
\bar{A}_{1}=2(q-1)^{2}\left(2 \pi / C_{1}\right)^{1 / 2} .
$$

Proof. We decompose (using $\lambda_{n-m, n}=-\lambda_{m, n}$ and $\lambda_{1,2}=0$ )

$$
p^{(2 N)}(o, o)=\sum_{n=3}^{\infty} \sum_{\substack{m=1 \\ \operatorname{gcd}(m, n)=1}}^{n-1} \mu\left(\lambda_{m, n}\right) \lambda_{m, n}^{2 N}=S_{1}(N)+S_{2}(N)
$$

where

$$
S_{1}(N)=2 \sum_{n=3}^{\infty} \mu\left(\lambda_{1, n}\right) \lambda_{1, n}^{2 N} \quad \text { and } \quad S_{2}(N)=\sum_{n=4}^{\infty} \sum_{\substack{m=2 \\ \operatorname{gcd}(m, n)=1}}^{n-2} \mu\left(\lambda_{m, n}\right) \lambda_{m, n}^{2 N} .
$$

Case $r>q$. Then, for $n \rightarrow \infty$,

$2 \mu\left(\lambda_{1, n}\right)=2 \pi^{2} A_{0} n^{-3} q^{-n}\left(1+O\left(n^{-2}\right)\right)$ where $A_{0}=2 q r(q+r)(q-1)(r-1) /(r-q)^{3}$.

Therefore, using Lemma 4,

$$
S_{1}(N)=A_{0} \rho(P)^{2 N} \Sigma(N ; 1,-3)
$$

has the asymptotic behavior that we have proposed for $p^{(2 N)}(o, o)$. Thus, it remains to show that $S_{2}(N)$ is dominated by $S_{1}(N)$. Note that for $2 \leq m \leq n-2$, we have

$$
\lambda_{m, n}^{2} \leq \rho(P)^{2} \cos ^{2} \frac{2}{n} \pi,
$$

and

$$
\sum_{\substack{m=2 \\ \operatorname{gcd}(m, n)=1}}^{n-2} \mu\left(\lambda_{m, n}\right) \leq A_{0}\left(\log \left(1-r^{-n}\right)-\log \left(1-q^{-n}\right)\right)=A_{0}\left(1+\varepsilon_{n}\right) q^{-n},
$$

where $\varepsilon_{n} \rightarrow 0$ as $n \rightarrow \infty$. Therefore,

$$
S_{2}(N) \leq A_{0} \rho(P)^{2 N} \Sigma(N ; 2,0) .
$$

Since $B_{2}>B_{1}$, Lemma 4 shows that $\Sigma(N ; 2,0) / \Sigma(N ; 1,-3) \rightarrow 0$, and comparing (5.3) with (5.4), we see that $S_{2}(N) / S_{1}(N) \rightarrow 0$ as $N \rightarrow \infty$.

Case $r=q$. The proof is basically the same. The only difference is that in this case, Theorem 1 yields

$$
\mu\left(\lambda_{1, n}\right) \sim(q-1)^{2} q^{-n},
$$

while in case $r>q$, we had the additional factor $n^{-3}$. Therefore

$$
S_{1}(N)=2(q-1)^{2} \Sigma(N ; 1,0) \quad \text { and } \quad S_{2}(N) \leq(q-1)^{2} \Sigma(N ; 2,1),
$$

whence Lemma 4 implies the result. 
It is quite surprising that the polynomial terms $N^{-5 / 6}$ versus $N^{1 / 6}$ are different when $r \neq q$, respectively $r=q$. The asymptotics in case $r=q$ were computed previously by Revelle [23] for a very similar random walk on the lamplighter group: In terms of $D L(q, r)$ this is SRW on the graph obtained by adding edges in the first tree $\left(\mathbb{T}_{q}\right)$, so that each vertex is connected to each of the siblings of its predecessor and then taking the horocyclic product as before. It turns out that for $q=r$, the $N$-step return probabilities of that random walk are just $2 p^{(N)}(o, o)$, see [29], Section 5 for details. The specific computations of [23] are very similar to ours, although [23] does not use the Plancherel measure.

\section{Plancherel Measure and Cumulative Spectral Measure}

Let $(X, o)$ and $\left(X^{\prime}, o^{\prime}\right)$ be locally finite, infinite graphs with respective roots $o$ and $o^{\prime}$. Let $R\left(X, X^{\prime}\right)$ be the largest radius $R$ for which there is a root-preserving isomorphism between the balls $B_{X}(o, R)$ and $B_{X^{\prime}}\left(o^{\prime}, R\right)$ in the respective graph metrics. A sequence of rooted graphs $\left(X_{n}, o_{n}\right)$ is said to converge to the graph $(X, o)$, if $R\left(X, X_{n}\right) \rightarrow \infty$. In this situation, given vertices $x, y \in X$, we can consider them via the respective isomorphisms as elements of $X_{n}$ for all $n \geq n(x, y)$, and we have the corresponding spectral measures $\mu_{x, y}^{(n)}$ associated with the SRW operator $P_{n}$ on $X_{n}$, as well as the measure $\mu_{x, y}$ associated with $P$ on $X$. (In particular, we consider $o$ as the common root of all graphs in the sequence.)

In this setting, it is a well known fact regarding operator convergence that

$$
\mu_{x, y}^{(n)} \rightarrow \mu_{x, y} \quad \text { weakly, as } n \rightarrow \infty
$$

see e.g., Grigorchuk and Zuk [13]. The main interest here is in the diagonal elements $\mu_{x, x}^{(n)} \rightarrow \mu_{x, x}$, in particular, in the case when $X$ is vertex-transitive, and $\mu_{x, x}=\mu_{o, o} \forall x \in$ $X$.

When the $X_{n}$ are finite graphs, another type of spectral measure is of interest in the place of $\mu_{o, o}^{(n)}$, namely the cumulative spectral measure

$$
\tilde{\mu}_{X_{n}}=\tilde{\mu}_{n}=\frac{1}{\left|X_{n}\right|} \sum_{x \in X_{n}} \mu_{x, x}^{(n)}=\frac{1}{\left|X_{n}\right|} \sum_{\lambda \in \operatorname{spec}\left(P_{n}\right)} \operatorname{mult}(\lambda) \delta_{\lambda},
$$

where $\operatorname{mult}(\lambda)$ is the multiplicity of $\lambda$ as an eigenvalue of $P_{n}$. If $X_{n}$ is vertex-transitive then $\tilde{\mu}_{n}=\mu_{o, o}^{(n)}$. In general, the following two questions are of interest.

(a) Does the sequence $\left(\tilde{\mu}_{n}\right)$ converge weakly to some probability measure $\tilde{\mu}$ ?

(b) Is $\tilde{\mu}=\mu_{o, o}$ ?

In case of a positive answer to question (6.2) (a), we call $\tilde{\mu}$ the cumulative spectral measure of $(X, o)$ with respect to the sequence $\left(X_{n}, o\right)$. Recently, the names Von Neumann and Serre have been associated with that measure.

All this applies, in particular, when each $X_{n}$ is an induced subgraph of $X$. ("Induced" means that when $x, y \in X_{n}$ are neighbors in $X$, then also $x \sim y$ in $X_{n}$.) In this situation, it may have advantages to use instead of $P_{n}$ the restriction (truncation) $P_{\mid n}=\left.P\right|_{X_{n}}$ of $P$ on $X_{n}$. Note that $P_{n}$ and $P_{\mid n}$ coincide in the interior of $X_{n}$, while they differ in the points of the boundary $\partial X_{n}$ of $X_{n}$ (i.e., the points of $X_{n}$ having a neighbor in $X \backslash X_{n}$ ), where $P_{\mid n}$ is strictly substochastic. The operator $P_{\mid n}$ acts on the same $\ell^{2}$-space as $P$, while $P_{n}$ uses different weights (vertex degrees) at the boundary points. The spectral measures of $P_{\mid n}$ 
also converge weakly to the respective spectral measures of $X$, and one can as well study the cumulative spectral measures $\tilde{\mu}_{\mid n}$ associated with $P_{\mid n}$ and their possible limit, again denoted $\tilde{\mu}$.

We remark that in the literature, the distinction between Plancherel (Kesten) and cumulative spectral measures has not always been very clear.

Question (6.2) has first been dealt with explicitly by McKay [19], who showed that when the $X_{n}$ are $(q+1)$-regular graphs with (asymptotically) few cycles, then the sequence $\tilde{\mu}_{n}$ converges weakly to the Plancherel measure of the tree $\mathbb{T}_{q}$. A systematic answer to question (6.2) (a) is given by Serre [26]. In [14], the spectrum of SRW on the lamplighter group $\mathbb{Z}_{2}$ Z (i.e., $D L(2,2)$ ) is computed via an approximating sequence of Schreier graphs, and it is shown that the corresponding cumulative spectral measure coincides with $\mu_{o, o}$.

The sequence $\left(X_{n}\right)$ of subsets of $X$ is called a Folner sequence, if $\inf _{n}\left|\partial X_{n}\right| /\left|X_{n}\right|=$ 0 . It is called expanding, if that infimum is positive. Recall that a graph is called amenable, if it has a Følner sequence. By Dodziuk [10], this holds if and only if the spectral radius of SRW satisfies $\rho(P)=1$. This notion comes from group theory; a group is called amenable, if it carries a finitely additive, left-invariant probability measure, and a finitely generated group is amenable if and only if one (equivalently, each) of its Cayley graphs with respect to a finite, symmetric generating set is amenable. For more details in the context of random walks, see e.g., [28], Section 10 and Section 12.

Theorem 3. Let $X$ be an infinite, connected locally finite vertex-transitive graph, and $\left(X_{n}\right)$ an increasing subsequence of finite subgraphs whose union is $X$.

(a) If $\left(X_{n}\right)$ is a Folner sequence then both $\tilde{\mu}_{n}$ and $\tilde{\mu}_{\mid n}$ converge weakly to the Plancherel measure of $X$.

(b) If $\left(X_{n}\right)$ is an expanding sequence then $\tilde{\mu}_{\mid n}$ does not converge to the Plancherel measure of $X$.

Proof. All involved measures are probability measures on $\mathbb{R}$ with compact support. Therefore, weak convergence holds if and only if for each $N \in \mathbb{N}$, the $N$-th moment of $\tilde{\mu}_{n}$ (resp. $\tilde{\mu}_{\mid n}$ ) converges to the $N$-th moment of the Plancherel measure. The latter is $M_{N}\left(\mu_{o, o}\right)=p^{(N)}(o, o)=p^{(N)}(x, x)$ for all $x \in X$ (by transitivity), while

$$
M_{N}\left(\tilde{\mu}_{n}\right)=\frac{1}{\left|X_{n}\right|} \sum_{x \in X_{n}} p_{X_{n}}^{(N)}(x, x)
$$

(analogously for $\tilde{\mu}_{\mid n}$ ). Now consider

$$
\partial_{N} X_{n}=\left\{x \in X_{n}: d\left(x, \partial X_{n}\right) \leq N / 2\right\} .
$$

If $x \in X_{n} \backslash \partial_{N} X_{n}$ then $p_{X_{n}}^{(N)}(x, x)=p_{\mid X_{n}}^{(N)}(x, x)=p^{(N)}(x, x)$. Therefore

$$
\begin{aligned}
M_{N}\left(\mu_{o, o}\right)-M_{N}\left(\tilde{\mu}_{\mid n}\right) & =\frac{1}{\left|X_{n}\right|} \sum_{x \in X_{n}}\left(p^{(N)}(x, x)-p_{\mid X_{n}}^{(N)}(x, x)\right) \\
& =\frac{1}{\left|X_{n}\right|} \sum_{x \in \partial_{N} X_{n}}\left(p^{(N)}(x, x)-p_{\mid X_{n}}^{(N)}(x, x)\right) .
\end{aligned}
$$

Now, if $\left(X_{n}\right)$ is a Følner sequence then

$$
\left|M_{N}\left(\mu_{o, o}\right)-M_{N}\left(\tilde{\mu}_{\mid n}\right)\right| \leq 2\left|\partial_{N} X_{n}\right| /\left|X_{n}\right| \rightarrow 0 \quad(n \rightarrow \infty),
$$


and the same holds for $\tilde{\mu}_{n}$. This proves (a).

To see (b), first note that $p^{(N)}(x, x)-p_{\mid X_{n}}^{(N)}(x, x)>0$ for every $x \in \partial X_{n}$. The involved transition probabilities regard only what happens in the ball $B(x, N / 2)$, and the restriction to $X_{n}$ means that only a part of that ball is admitted for the walker, while the rest is taboo. By transitivity, all these balls are isomorphic, and up to isometry, there are only finitely many ways to subdivide a ball into the admitted and taboo parts. Hence, as $x$ varies, while $N$ is fixed, there are only finitely many different values of $p_{\mid X_{n}}^{(N)}(x, x)$. Consequently, there is $\varepsilon_{N}>0$ such that

$$
p^{(N)}(x, x)-p_{\mid X_{n}}^{(N)}(x, x) \geq \varepsilon_{N} \quad \forall n \in \mathbb{N}, x \in \partial X_{n} .
$$

Therefore

$$
M_{N}\left(\mu_{o, o}\right)-M_{N}\left(\tilde{\mu}_{\mid n}\right) \geq \varepsilon_{N}\left|\partial X_{n}\right| /\left|X_{n}\right|,
$$

which does not tend to zero as $n \rightarrow \infty$.

Remark 1. (1) Theorem 3 is of course also valid for the adjacency matrix of $X$ and its restriction to $X_{n}$, acting on $\ell^{2}(X)$, resp. $\ell^{2}\left(X_{n}\right) \subset \ell^{2}(X)$.

(2) Part (a) can also be formulated for a sequence of finite graphs $X_{n}$ converging to $X$ that are not necessarily subgraphs of $X$. In that case, define

$$
\partial^{N} X_{n}=\left\{x \in X_{n}: B_{X_{n}}(x, N / 2) \text { is not isomorphic with } B_{X}(o, N / 2)\right\} .
$$

Then we call $\left(X_{n}\right)$ a Folner approximation of $X$ if $\left|\partial^{N} X_{n}\right| /\left|X_{n}\right| \rightarrow 0$ as $n \rightarrow \infty$ for every $N \in \mathbb{N}$.

This condition requires that $X$ is vertex-transitive. If $\left(X_{n}\right)$ is a $F \varnothing$ lner approximation of $X$, then the above argument shows that the cumulative spectral measures of $X_{n}$ converge weakly to the Plancherel measure of $X$.

(3) As mentioned above, Grigorchuk and Żuk [14] consider $\mathbb{Z}_{2} 2 \mathbb{Z}$, i.e., $D L(2,2)$, and use an approximation by Schreier graphs and the associated cumulative spectral measures. They show that the latter converge to the Plancherel measure. This can also be interpreted in terms of a Følner approximation. Indeed, the graphs $X_{n}$ defined by the action of the automaton considered in [14] can be defined alternatively as follows: Let $m$ be the smallest power of 2 that is $\geq n$, and consider the group $\Gamma_{n}=\mathbb{Z}_{2}^{n} \rtimes \mathbb{Z}_{m}$, with $\mathbb{Z}_{m}$ acting as a matrix with 1's on the diagonal and just above, 0 's elsewhere. This is the permutation group acting on level $n$. The generator $a$ generates $\mathbb{Z}_{m}$, and $b=\sigma a$ where $\sigma$ is any non-trivial element of $\mathbb{Z}_{2}^{m}$. The Schreier graph is the homogeneous space $X_{n}=\mathbb{Z}_{m} \backslash \Gamma_{n}$.

The elements in $X_{n}$ can be naturally identified with vectors in $\mathbb{Z}_{2}^{n}$. Fix an integer $N$, and let $Y_{n}$ denote those vectors that are not periodic of period less than $N$. On one hand, $\left|Y_{n}\right| /\left|X_{n}\right| \rightarrow 1$ as $n \rightarrow \infty$, and on the other hand, any ball of radius $N$ around $y \in Y_{n}$ embeds in the lamplighter group $\mathbb{Z}_{2}^{\infty} \rtimes \mathbb{Z}$; therefore the graphs $X_{n}$ have the Følner approximation property.

Our computation of the spectrum of SRW on $D L(q, r)$ in Section 3 is linked with tetrahedra. In this context, it is natural to take for $X_{n}$ an increasing family of tetrahedra $S_{n}$ with height $n \rightarrow \infty$, whose union is $D L$, and consider the associated cumulative measures $\tilde{\mu}_{\mid n}$.

If $r=q$, i.e., for the lamplighter group, we have $\left|S_{n}\right|=(n+1) q^{n}$ and $\left|\partial S_{n}\right|=2 q^{n}$. Therefore $\left(S_{n}\right)$ is a very natural Følner sequence in $\operatorname{DL}(q, q)$, which is indeed a Cayley graph of an amenable group. 
On the other hand, if $r>q$ then $\left|S_{n}\right| \sim r^{n+1} /(r-q)$ and $\left|\partial S_{n}\right| \sim r^{n}$, as $n \rightarrow \infty$, whence $\left(S_{n}\right)$ is an expanding sequence. We know that in this case $D L(q, r)$ is a nonamenable graph [25].

Corollary 3. If $r=q$ then for $S_{n}$, both sequences $\tilde{\mu}_{n}$ and $\tilde{\mu}_{\mid n}$ converge weakly to the Plancherel measure of $D L(q, q)$.

If $r \neq q$ then $\tilde{\mu}_{\mid n}$ does not converge to the Plancherel measure of $D L(q, r)$.

We remark that we did not compute the actual limit of $\tilde{\mu}_{\mid n}$, when $r \neq q$. This can be done along the lines of the following computations.

Let us now consider the sequence $\tilde{\mu}_{n}$ corresponding to SRW on the graphs $S_{n}$ for $r \neq q$.

We shall always suppose that $r>q$.

Let $S=S\left(a_{1}, a_{2}\right)$ be any tetrahedron with height $n$. We want to compute the cumulative spectral measure of SRW $P_{S}$ on $S$. We define

$$
\partial^{1} S=\left\{b_{1} a_{2}: b_{1} \in \mathbb{T}_{q}, b_{1} a_{2} \in D L\right\} \quad \text { and } \quad \partial^{2} S=\left\{a_{1} b_{2}: b_{2} \in \mathbb{T}_{r}, a_{1} b_{2} \in D L\right\}
$$

(the lower and upper parts of $\partial S$ ). Then $P_{S}$ coincides with $P$ in the interior $S \backslash \partial S$ of $S$, while

$$
\begin{array}{ll}
p_{S}\left(b_{1} a_{2}, x_{1} x_{2}\right)=1 / r & \text { if } \quad b_{1} a_{2} \in \partial^{1} S, x_{1}=b_{1}^{-}, x_{2}^{-}=a_{2}, \quad \text { and } \\
p_{S}\left(a_{1} b_{2}, x_{1} x_{2}\right)=1 / q & \text { if } \quad a_{1} b_{2} \in \partial^{2} S, x_{1}^{-}=a_{1}, x_{2}=b_{2}^{-} .
\end{array}
$$

In order to compare with $P$ acting on $\ell^{2}(X)$, it is more natural to consider $P_{S}$ as a self-adjoint operator on $\ell^{2}\left(S, \mathrm{~m}_{S}\right)$, where

$$
\mathrm{m}_{S}\left(x_{1} x_{2}\right)=\left\{\begin{array}{lll}
1, & \text { if } & x_{1} x_{2} \in S \backslash \partial S \\
r /(r+q), & \text { if } & x_{1} x_{2} \in \partial^{1} S \\
q /(r+q), & \text { if } & x_{1} x_{2} \in \partial^{2} S
\end{array}\right.
$$

instead of using the reference measure $\operatorname{deg}_{S}=(q+r) \mathrm{m}_{S}$.

We already know part of the spectrum of $P_{S}$, namely, the spectrum of $P_{S}$ acting on the space of all horizontal functions with support in $S$. We need further eigenfunctions besides the horizontal ones. Recall the functions $f_{k}^{[S, i, j]}$ and $g_{m}^{[S, i, j]}$ constructed in (3.8), $i=1, \ldots, q-1, j=1, \ldots, r-1$, with $k, m=1, \ldots, n-1$. We can also include $k=0, n$ with $f_{0}^{[S, i, j]}=f_{n}^{[S, i, j]}=0$. We shall now extend the range of $i$ and $j$, adding also the values $i=0$ and $j=0$. Namely, in analogy with (3.3) and (3.4), and using (3.7), we define for $k=0, \ldots, n$ and $x=x_{1} x_{2} \in S$

$$
\begin{aligned}
f_{k}^{[S, 0,0]}\left(x_{1} x_{2}\right) & = \begin{cases}q^{-k / 2} r^{-(n-k) / 2} & \text { if } x_{1} \in H_{\mathfrak{h}\left(a_{1}\right)+k}^{1}, \\
0 & \text { otherwise },\end{cases} \\
f_{k}^{[S, 0, j]}\left(x_{1} x_{2}\right) & = \begin{cases}q^{-k / 2} f_{n-k}^{2}\left[\varphi_{j}^{2}\right]\left(x_{2}\right) & \text { if } x_{1} \in H_{\mathfrak{h}\left(a_{1}\right)+k}^{1}, \\
0 & \text { otherwise } \quad(j=1, \ldots, r-1),\end{cases} \\
f_{k}^{[S, i, 0]}\left(x_{1} x_{2}\right) & = \begin{cases}f_{k}^{1}\left[\varphi_{i}^{1}\right]\left(x_{1}\right) r^{-(n-k) / 2} & \text { if } x_{1} \in H_{\mathfrak{h}\left(a_{1}\right)+k}^{1}, \\
0 & \text { otherwise } \quad(i=1, \ldots, q-1) .\end{cases}
\end{aligned}
$$


The different functions $f_{k}^{[S, i, j]}$ are all orthogonal, and when $k \in\{1, \ldots, n-1\}$, then they have norm one. On the other hand,

$$
\left\|f_{0}^{[S, 0, j]}\right\|^{2}=\frac{q}{r+q} \quad \text { and } \quad\left\|f_{0}^{[S, i, 0]}\right\|^{2}=\frac{r}{r+q} \quad \text { in } \quad \ell^{2}\left(S, \mathrm{~m}_{S}\right) .
$$

As usual, we also think of all functions of (6.4) as being extended to the whole of $D L$, with value 0 outside of $S$. It is also convenient to set $f_{k}^{[S, i, j]} \equiv 0$ when $k<0$ or $k>n$. Let $\rho=\rho(P)=\frac{2 \sqrt{q r}}{q+r}$. One has for all pairs $(i, j)$

$$
P_{S} f_{k}^{[S, i, j]}=\frac{\rho}{2}\left(f_{k-1}^{[S, i, j]}+f_{k+1}^{[S, i, j]}\right) \begin{cases}\forall k \in \mathbb{Z}, & \text { if } i, j \neq 0, \\ \forall k \in \mathbb{Z} \backslash\{ \pm 1\}, & \text { if } i=0, j \neq 0, \\ \forall k \in \mathbb{Z} \backslash\{n \pm 1\}, & \text { if } i \neq 0, j=0, \\ \forall k \in \mathbb{Z} \backslash\{ \pm 1, n \pm 1\}, & \text { if } i=j=0,\end{cases}
$$

$P_{S} f_{1}^{[S, 0, j]}=\frac{\sqrt{r}}{\sqrt{q}} f_{0}^{[S, 0, j]}+\frac{\rho}{2} f_{2}^{[S, 0, j]}, \quad$ and

$P_{S} f_{n-1}^{[S, i, 0]}=\frac{\rho}{2} f_{n-2}^{[S, i, 0]}+\frac{\sqrt{q}}{\sqrt{r}} f_{n}^{[S, i, 0]}$.

Case 1. $i, j \neq 0$. In this case, (6.6) was stated in Lemma 2, and we find the corresponding eigenvalues and eigenfunctions as in Section 3.

Case 2. $i=j=0$. None of $f_{0}^{[S, 0,0]}$ and $f_{n}^{[S, 0,0]}$ vanish. The action of $P_{S}$ on the space spanned by $f_{k}^{[S, 0,0]}(k=0, \ldots, n)$ is described by the $(n+1) \times(n+1)$-matrix

$$
M_{n+1}^{o}=\frac{\rho}{2}\left(\begin{array}{cccccc}
0 & 1 & & & & \\
\frac{q+r}{q} & \ddots & \ddots & & & \\
& 1 & \ddots & \ddots & & \\
& & \ddots & \ddots & 1 & \\
& & & \ddots & \ddots & \frac{q+r}{r} \\
& & & & 1 & 0
\end{array}\right)
$$

over the indices $k=0, \ldots, n$. In general, if we have an eigenvalue $\lambda$ of $M_{n+1}^{o}$ and an associated left eigenvector $\psi$, written as a function on $\{0, \ldots, n\}$, then we obtain a normalized eigenfunction $g$ of $P_{S}$ and its norm in $\ell^{2}\left(S, \mathrm{~m}_{S}\right)$ by setting

$g=C \sum_{k=0}^{n} \psi(k) f_{k}^{[S, 0,0]}, \quad$ where $\quad C^{2}=\frac{q}{r+q} \psi(0)^{2}+\sum_{k=1}^{n-1} \psi(k)^{2}+\frac{r}{r+q} \psi(n)^{2}$

[using (6.5)], as in Section 3. Applying this recipe, we start with the following eigenvalues and eigenfunctions of $M_{n+1}^{o}$, resp. $P_{S}$.

$\begin{array}{lll}\lambda_{0, n}=1, & \psi_{0, n}(k)=\sqrt{q / r}^{k}, & g_{0}^{[S, 0,0]}(x)=\left(2 q r r^{r^{n}-q^{n}}\right)^{-1 / 2}, \quad \text { and } \\ \lambda_{n, n}=-1, & \psi_{n, n}(k)=(-\sqrt{q / r})^{k}, & g_{n}^{[S, 0,0]}(x)=(-1)^{\mathfrak{h}\left(x_{1}\right)}\left(2 q r \frac{r^{n}-q^{n}}{r^{2}-q^{2}}\right)^{-1 / 2},\end{array}$ 
where $x \in S$. Next, for $m=1, \ldots, n-1$, set

$$
\beta_{m, n}^{o}=\arctan \left(\frac{q+r}{q-r} \tan \frac{m}{n} \pi\right) .
$$

Some computations yield the same eigenvalue as in (3.6) and the following corresponding eigenfunction of $P_{S}$ :

$$
\lambda_{m, n}=\rho \cos \frac{m}{n} \pi, \quad g_{m}^{[S, 0,0]}=\sum_{k=0}^{n} \frac{\sqrt{2}}{\sqrt{n}} \sin \left(\frac{k m}{n} \pi+\beta_{m, n}^{o}\right) f_{k}^{[S, 0,0]} .
$$

Case 3. $i=0, j \neq 0$. Then $f_{0}^{[S, 0, j]}$ does not vanish, while $f_{n}^{[S, 0, j]} \equiv 0$. The action of $P_{S}$ on the space spanned by $f_{k}^{[S, 0, j 0]}(k=0, \ldots, n-1)$ is described by the $(n \times n)$-matrix

$$
M_{n}^{\prime}=\frac{\rho}{2}\left(\begin{array}{ccccc}
0 & 1 & & & \\
\frac{q+r}{q} & \ddots & \ddots & & \\
& 1 & \ddots & \ddots & \\
& & \ddots & \ddots & 1 \\
& & & 1 & 0
\end{array}\right)
$$

over the indices $k=0, \ldots, n-1$. Computations are slightly more involved in this case; we present the results.

Case 3.A. $n \geq \frac{r+q}{r-q}$.

A.l If $n>\frac{r+q}{r-q}$ then there is precisely one $\alpha=\alpha_{0, n}>0$ that solves the equation

$$
e^{2 n \alpha}=\frac{q-r e^{2 \alpha}}{q e^{2 \alpha}-r} .
$$

(If $n \leq \frac{r+q}{r-q}$ then there is no such solution.) We get

$$
\begin{gathered}
\lambda_{0, n}^{\prime}=\rho \cosh \alpha_{0, n}, \quad g_{0}^{[S, 0, j]}=C_{0, n}^{\prime} \sum_{k=0}^{n-1} \sinh \left((n-k) \alpha_{0, n}\right) f_{k}^{[S, 0, j]} \\
\lambda_{n-1, n}^{\prime}=-\rho \cosh \alpha_{0, n}, \quad g_{n-1}^{[S, 0, j]}=C_{n-1, n}^{\prime} \sum_{k=0}^{n-1}(-1)^{k} \sinh \left((n-k) \alpha_{0, n}\right) f_{k}^{[S, 0, j]} . \\
A .2 \text { If } n=\frac{r+q}{r-q} \text { then we find, setting } \alpha_{0, n}=0 \text { and } \alpha_{n-1, n}=\pi, \\
\lambda_{0, n}^{\prime}=\rho=\rho \cos \alpha_{0, n}, \quad g_{0}^{[S, 0, j]}=C_{0, n}^{\prime} \sum_{k=0}^{n-1}\left(1-\frac{k}{n}\right) f_{k}^{[S, 0, j]} \\
\lambda_{n-1, n}^{\prime}=-\rho=\rho \cos \alpha_{n-1, n}, \quad g_{n-1}^{[S, 0, j]}=C_{n-1, n}^{\prime} \sum_{k=0}^{n-1}(-1)^{k}\left(1-\frac{k}{n}\right) f_{k}^{[S, 0, j]} .
\end{gathered}
$$


In both subcases A.1 and A.2, the equation

$$
\cot (n \alpha)=\frac{r-q}{r+q} \cot \alpha, \quad \alpha \in(0, \pi)
$$

has exactly $n-2$ distinct solutions $\alpha_{m, m}, m=1, \ldots, n-2$. For each $\alpha_{m, n}$, we get

$$
\lambda_{m, n}^{\prime}=\rho \cos \alpha_{m, n}, \quad g_{m}^{[S, 0, j]}=C_{m, n}^{\prime} \sum_{k=0}^{n-1} \sin \left((n-k) \alpha_{m, n}\right) f_{k}^{[S, 0, j]} .
$$

In (6.11), (6.12), and (6.14), the normalizing constants $C_{m, n}^{\prime}$ are computed as $C$ in (6.7).

Case 3.B. $n<\frac{r+q}{r-q}$.

In this case, the Equation (6.13) has exactly $n$ distinct solutions $\alpha_{m, n}, m=0, \ldots, n-$ 1. Associated with each of those $\alpha_{m, n}$ there is a solution of the form (6.14).

Case 4. $i \neq 0, j=0$. Then $f_{n}^{[S, i, 0]}$ does not vanish, while $f_{0}^{[S, i, 0]} \equiv 0$. The action of $P_{S}$ on the space spanned by $f_{k}^{[S, i, 0]}(k=1, \ldots, n)$ is described by the $(n \times n)$-matrix

$$
M_{n}^{\prime \prime}=\frac{\rho}{2}\left(\begin{array}{ccccc}
0 & 1 & & & \\
1 & \ddots & \ddots & & \\
& \ddots & \ddots & 1 & \\
& & \ddots & \ddots & \frac{q+r}{r} \\
& & & 1 & 0
\end{array}\right)
$$

over the indices $k=1, \ldots, n$. This is analogous to Case 3.B, exchanging $q$ with $r$ and $k, m$ with $n-k, m-k$. The equation

$$
\cot (n \gamma)=-\frac{r-q}{r+q} \cot \gamma, \quad \gamma \in(0, \pi)
$$

has exactly $n$ distinct solutions $\gamma_{m, n}, m=1, \ldots, n$. Associated with each of them, we find

$$
\lambda_{m, n}^{\prime \prime}=\rho \cos \gamma_{m, n}, \quad g_{m}^{[S, i, 0]}=C_{m, n}^{\prime \prime} \sum_{k=1}^{n} \sin \left(k \gamma_{m, n}\right) f_{k}^{[S, i, 0]}
$$

with normalizing constant $C_{m, n}^{\prime \prime}$ according to (6.7).

We set

$$
\begin{aligned}
\mathcal{A}_{S}^{o} & =\left\{g_{m}^{[S, 0,0]}: m=0, \ldots, n\right\} \\
\mathcal{A}_{S}^{\prime} & =\left\{g_{m}^{[S, 0, j]}: m=0, \ldots, n-1, j=1, \ldots, r-1\right\}, \\
\mathcal{A}_{S}^{\prime \prime} & =\left\{g_{m}^{[S, i, 0]}: m=1, \ldots, n, i=1, \ldots, q-1\right\} .
\end{aligned}
$$

Lemma 5. The orthogonal complement of the subspace of horizontal functions in $\ell^{2}(S)$, where $S=S\left(a_{1}, a_{2}\right)$, is spanned by

$$
\mathcal{A}_{S}=\mathcal{A}_{S}^{o} \cup \bigcup\left\{\mathcal{A}_{S\left(a_{1}, c_{2}\right)}^{\prime}: S\left(a_{1}, c_{2}\right) \subseteq S\right\} \cup \bigcup\left\{\mathcal{A}_{S\left(c_{1}, a_{2}\right)}^{\prime \prime}: S\left(c_{1}, a_{2}\right) \subseteq S\right\},
$$

where $c_{1}$ and $c_{2}$ vary. The functions in $\mathcal{A}_{S}$ are all orthonormal with respect to each other. 
Proof. Orthonormality follows from the straightforward verification that this is true for the corresponding functions $f_{m}$ in the place of the $g_{m}$. To prove that $\mathcal{A}_{S}$ spans the orthogonal complement of horizontal functions, we proceed as in the proof of Proposition 1 and consider the $k$-th level $L_{k}$ of $S$. The space of functions supported in $L_{k}$ has dimension $q^{k} r^{n-k}$, whence the codimension of the space of horizontal functions supported in $L^{k}$ is $q^{k}+r^{n-k}-1$. Direct counting shows that this is precisely the number of functions $f_{m}^{\left[S\left(c_{1}, c_{2}\right), i, j\right]}$ with $S\left(c_{1}, c_{2}\right) \subseteq S, c_{1}=a_{1}$ or $c_{2}=a_{2}$, and $i=0$ or $j=0$, that do not vanish on $L_{k}$.

Thus, our long computations lead to the following result, which also shows that $\tilde{\mu}_{n}$ does not converge to the Plancherel measure when $r \neq q$.

Proposition 3. If $r>q$, then the cumulative spectral measures $\tilde{\mu}_{n}$ associated with (non-truncated) $S R W$ on $S_{n}$ converge weakly to the measure $\tilde{\mu}+\tilde{v}$, given as follows.

$$
\begin{aligned}
\tilde{\mu}= & (q-1)(r-1) \sum_{N=2}^{\infty} r^{-N} \sum_{m=1}^{N-1} \delta_{\lambda_{m, N}}+(r-q)(r-1) \sum_{2 \leq N \leq \frac{r+q}{r-q}} r^{-N-1} \sum_{m=0}^{N-1} \delta_{\lambda_{m, N}^{\prime}} \\
& +(r-q)(r-1) \sum_{N>\frac{r+q}{r-q}} r^{-N-1} \sum_{m=1}^{N-2} \delta_{\lambda_{m, N}^{\prime}}
\end{aligned}
$$

with $\lambda_{m, N}=\rho(P) \cos \left(\frac{m}{M} \pi\right)$ and $\lambda_{m, N}^{\prime}=\rho(P) \cos \alpha_{m, N}$ given by (6.13).

$$
\tilde{v}=(r-q)(r-1) \sum_{N>\frac{r+q}{r-q}} r^{-N-1}\left(\delta_{\lambda_{0, N}^{\prime}}+\delta_{-\lambda_{0, N}^{\prime}}\right)
$$

with $\lambda_{0, N}^{\prime}=\rho(P) \cosh \alpha_{0, N}$ given by (6.10).

The support of the measure $\tilde{\mu}$ is the interval $[-\rho(P), \rho(P)]$.

The support $\left\{ \pm \lambda_{0, N}^{\prime}: N>\frac{r+q}{r-q}\right\}$ of $\tilde{v}$ is contained in $[-1,-\rho(P)] \cup[\rho(P), 1]$. The sequence $\left(\lambda_{0, N}^{\prime}\right)$ is strictly increasing with limit 1 .

Proof. First of all, $\left|S_{n}\right|=\left(r^{n+1}-q^{n+1}\right) /(r-q)$.

If we fix $n$ and consider $N \in\{2, \ldots, n\}$, then $S_{n}=S\left(a_{1}, a_{2}\right)$ contains $\left(r^{n-N+1}-\right.$ $\left.q^{n-N+1}\right) /(r-q)$ different tetrahedra $S\left(c_{1}, c_{2}\right)$ with height $N$. With each of those, and each $i \in\{1, \ldots, q-1\}$ and $j \in\{1, \ldots, r-1\}$, we associate each of the eigenvalues $\lambda_{m, N}$, $m=1, \ldots, N-1$. Thus, taking into account all those tetrahedra of height $N$, we count each $\lambda_{m, N}$ precisely $(q-1)(r-1)\left(r^{n-N+1}-q^{n-N+1}\right) /(r-q)$ times. If we divide by $\left|S_{n}\right|$ and let $n \rightarrow \infty$, we get the first of the three parts of $\tilde{\mu}$ (with an implicit use of dominated convergence).

Also, $S_{n}$ contains $r^{n-N}$ different tetrahedra $S\left(a_{1}, c_{2}\right)$ with height $N$, where only $c_{2}$ is allowed to vary. Associated with each of them, and with each $j \in\{1, \ldots, r-1\}$, we have each of the eigenvalues $\lambda_{m, N}^{\prime}, m=1, \ldots, N-1$. Again, taking into account all those tetrahedra, dividing by $\left|S_{n}\right|$, and letting $n \rightarrow \infty$, we obtain the second and third parts of $\tilde{\mu}$, plus $\tilde{v}$. The subdivision is according to whether $\left|\lambda_{m, N}^{\prime}\right| \leq \rho(P)$ or $>\rho(P)$, respectively.

The contributions to the spectrum of SRW on $S_{n}$ that come from $\mathcal{A}_{S\left(a_{1}, a_{2}\right)}^{o}$ and $\mathcal{A}_{S\left(c_{1}, a_{2}\right)}^{\prime \prime}$ (where $S\left(c_{1}, a_{2}\right) \subseteq S\left(a_{1}, a_{2}\right)$ and $c_{1}$ varies) vanish as $n \rightarrow \infty$, because $q^{n} / r^{n} \rightarrow 0$. 


\section{Final Observations}

A. Random walks with drift. Besides SRW on $D L(q, r)$, it may also be instructive to consider the following variant. If the actual position is $x=x_{1} x_{2} \in D L(q, r)$, then we first toss a coin, where "head" comes up with probability $\alpha \in(0,1)$. If head comes up, then we step at random to one among the $q$ neighbors $y=y_{1} y_{2}$ of $x$ with $y_{1}^{-}=x_{1}$ (i.e., downwards in Figure 2). Otherwise, we step at random to one among the $r$ neighbors $y$ of $x$ with $y_{2}^{-}=x_{2}$. Thus, we obtain the following generalization $P_{\alpha}$ of simple random walk on $D L(q, r)$. For $x=x_{1} x_{2}, y=y_{1} y_{2} \in D L(q, r)$

$$
p_{\alpha}(x, y)=\left\{\begin{array}{llll}
\alpha / q & \text { if } y_{1}^{-}=x_{1} & \text { and } & y_{2}=x_{2}^{-} \\
(1-\alpha) / r & \text { if } y_{1}=x_{1}^{-} & \text {and } & y_{2}^{-}=x_{2} \\
0 & \text { otherwise } & &
\end{array}\right.
$$

In order to interpret this in terms of a lamplighter when $r=q$, it is best to think of the lamps not placed at each vertex of the two-way-infinite path $\mathbb{Z}$, but at the middle of each edge. Each lamp may have $q$ different intensities or states ( $\equiv$ elements of $\mathbb{Z}_{q}$ ), the state "off" corresponding to $0 \in \mathbb{Z}_{q}$. Only finitely many lamps may be switched on. At each step, the lamplighter tosses his $\alpha$-coin. If "head" comes up, he moves "down" (from $k$ to $k+1$ ) and switches the lamp on the transversed edge to a random state. Otherwise, he moves "up" (to $k-1)$ and again switches the lamp on the transversed edge to a random state.

We remark that for all values $q, r$, the random walk $P_{\alpha}$ on $D L(q, r)$ may be interpreted as a lamplighter walk in an extended sense. Imagine that on each edge of $\mathbb{Z}$, there is a green lamp with $q$ possible intensities (including "off") plus a red lamp with $r$ possible intensities (including "off"). The rule is that only finitely many lamps may be switched on, and in addition, if the lamplighter stands at $k$, then all lamps between $k$ and $-\infty$ have to be in a green state, while all lamps between $k$ and $+\infty$ must be in a red state. The lamplighter tosses his $\alpha$-coin. If "head" comes up, he moves "down" (from $k$ to $k+1$ ) and switches the green lamp on the transversed edge to a random state, while switching off the red lamp on that edge. Otherwise, he moves "up" (to $k-1$ ) and switches the red lamp on the transversed edge to a random state, while switching off the green lamp on that edge.

SRW on $D L(q, r)$ is $P_{\alpha}$ with $\alpha=\frac{q}{q+r}$. In order to compare $D L(q, r)$ with $D L(q, q)$, it may be more natural to consider the same $\alpha$ in each case.

For arbitrary $\alpha$, we have

$$
m_{\alpha}(x) p_{\alpha}(x, y)=m_{\alpha}(y) p_{\alpha}(y, x), \quad \text { where } \quad m_{\alpha}(x)=\left(\frac{\alpha r}{(1-\alpha) q}\right)^{\mathfrak{h}\left(x_{1}\right)},
$$

i.e., $P_{\alpha}$ is $m_{\alpha}$-reversible. Therefore, $P_{\alpha}$ acts as a self-adjoint operator on the weighted space $\ell^{2}\left(D L, m_{\alpha}\right)$ with inner product

$$
\langle f, g\rangle_{\alpha}=\sum_{x} f(x) g(x) m_{\alpha}(x) .
$$

A quick computation shows that

$$
\frac{\sqrt{m_{\alpha}(x)}}{\sqrt{m_{\alpha}(y)}} p_{\alpha}(x, y)=t_{\alpha} p(x, y) \quad \forall x, y \in D L, \quad \text { where } \quad t_{\alpha}=\frac{\sqrt{4 \alpha(1-\alpha)}}{\rho(P)} .
$$

Here, $p(x, y)$ refers to simple random walk, and $\rho(P)=2 \sqrt{q r} /(q+r)$ is the spectral radius of the latter. This can be interpreted in terms of the Hilbert space isomorphism 
$T_{\alpha}: \ell^{2}\left(D L, m_{\alpha}\right) \rightarrow \ell^{2}(D L)$ (the latter with respect to the counting measure), where $T_{\alpha} f(x)=\sqrt{m_{\alpha}(x)} f(x)$. We find that

$$
T_{\alpha} P_{\alpha} f=t_{\alpha} \cdot P T_{\alpha} f \quad \forall f \in \ell^{2}\left(D L, m_{\alpha}\right) .
$$

Corollary 4. The spectrum of the operator $P_{\alpha}$ acting on $\ell^{2}\left(D L, m_{\alpha}\right)$ is the interval $[-\sqrt{4 \alpha(1-\alpha)}, \sqrt{4 \alpha(1-\alpha)}]$. It is obtained by dilating the spectrum of $P$ acting on $\ell^{2}(D L)$ (computed in Theorem 1) by the factor $t_{\alpha}$. The Plancherel measure associated with $P_{\alpha}$ is the image of the Plancherel measure associated with $P$ (computed in Corollary 2) under this dilation.

Corollary 5. The return probabilities of $P_{\alpha}$ behave asymptotically as follows.

(i) If $r>q$ then

$$
p^{(2 N)}(o, o) \sim A_{1}(4 \alpha(1-\alpha))^{N} \exp \left(-B_{1} N^{1 / 3}\right) N^{-5 / 6} \quad \text { as } \quad N \rightarrow \infty .
$$

(ii) If $r=q$ then

$$
p^{(2 N)}(o, o) \sim \bar{A}_{1}(4 \alpha(1-\alpha))^{N} \exp \left(-B_{1} N^{1 / 3}\right) N^{1 / 6} \quad \text { as } N \rightarrow \infty
$$

(The constants $A_{1}, \bar{A}_{1}$, and $B_{1}$ are as in Theorem 2 ).

This direct comparison of the $P_{\alpha}$ underlines how surprising it is that for $r>q$ (red and green lamps with different numbers of states) the asymptotics scale down by a factor of $N$ with respect to the case $r=q$ (only one type of lamps, or equivalently, red and green lamps with the same number of states).

We remark here that Bertacchi [3] has proved several basic results for general random walks on $D L(q, r)$, without being aware that they apply, in particular, to random walks on $\mathbb{Z}_{q} 2 \mathbb{Z}$. For example, when applied to $P_{\alpha}$, one gets a rate of escape theorem and a central limit theorem, as follows.

In $Z_{n}$ is the random vertex at time $n$ according to $P_{\alpha}$, with starting point $Z_{0}=o$, then

$$
\frac{d\left(Z_{n}, Z_{0}\right)}{n} \rightarrow|2 \alpha-1| \text { almost surely }
$$

If $\alpha \neq 1 / 2$ then

$$
\frac{d\left(Z_{n}, Z_{0}\right)-|2 \alpha-1| n}{\sqrt{4 \alpha(1-\alpha) n}} \rightarrow \mathcal{N}(0,1) \text { in law },
$$

where $\mathcal{N}(0,1)$ is the standard normal distribution. If $\alpha=1 / 2$ then

$$
\frac{d\left(Z_{n}, Z_{0}\right)}{\sqrt{n}} \rightarrow \mathcal{M} \text { in law },
$$

where $\mathcal{M}$ is the probability distribution on $\mathbb{R}^{+}$with density

$$
\frac{d \mathcal{M}}{d t}=\frac{1}{\sqrt{2 \pi}}\left(e^{-t^{2} / 4}-e^{-t^{2}}\right) \mathbf{1}_{[0, \infty)}(t) .
$$

Note that $2 \alpha-1$ and $4 \alpha(1-\alpha)$ are mean and variance (respectively) of the projected random variable $\widetilde{\Pi}\left(Z_{1}\right)$ on $\mathbb{Z}$ (see definition of $\widetilde{\Pi}$ a few lines below). Also note that these results, contrary to Corollary 5 , do not differ when $r=q$, resp. $r \neq q$. 
B. Projections. We have the natural projections $\Pi_{1}, \Pi_{2}$ and $\widetilde{\Pi}$ of $D L(q, r)$ onto $\mathbb{T}_{q}$ and $\mathbb{T}_{r}$, respectively, namely, $\Pi_{i}\left(x_{1} x_{2}\right)=x_{i}$ and $\widetilde{\Pi}\left(x_{1} x_{2}\right)=\mathfrak{h}\left(x_{1}\right)=\mathfrak{h} \circ \Pi_{1}(x)$. Associated with them, we have the projected random walks $P_{\alpha, q}, P_{1-\alpha, r}$ and $\widetilde{P}_{\alpha}$, where

$$
p_{\alpha, q}\left(x_{1}, y_{1}\right)=\left\{\begin{array}{ll}
\alpha / q, & \text { if } y_{1}^{-}=x_{1}, \\
1-\alpha, & \text { if } y_{1}=x_{1}^{-}, \\
0, & \text { otherwise. }
\end{array} \quad \text { and } \quad \tilde{p}_{\alpha}(k, l)= \begin{cases}\alpha, & \text { if } l=k+1, \\
1-\alpha, & \text { if } l=k-1, \\
0, & \text { otherwise } .\end{cases}\right.
$$

( $P_{1-\alpha, r}$ is analogous to $P_{\alpha, q}$.) The projections are compatible with these transition operators in the sense of factorization of Markov chains, i.e.,

$$
\begin{aligned}
p_{\alpha, q}\left(x_{1}, y_{1}\right) & =\sum_{\Pi_{1}(y)=y_{1}} p_{\alpha}(x, y) \text { for every } x \text { with } \Pi_{1}(x)=x_{1}, \quad \text { and } \\
\widetilde{p}(k, l) & =\sum_{\widetilde{\Pi}_{1}(y)=l} p_{\alpha}(x, y) \quad \text { for every } x \text { with } \widetilde{\Pi}(x)=k .
\end{aligned}
$$

Now, $P_{\alpha, q}$ is reversible with respect to $m_{\alpha, q}\left(x_{1}\right)=\left(\frac{\alpha}{q(1-\alpha)}\right)^{\mathfrak{h}\left(x_{1}\right)}$, where $x_{1} \in \mathbb{T}_{q}$. Also, $\widetilde{P}_{\alpha}$ is reversible with respect to $\tilde{m}_{\alpha}(k)=\left(\frac{\alpha}{(1-\alpha)}\right)^{k}$. These operators are self-adjoint on the respective spaces $\ell^{2}\left(\mathbb{T}_{q}, m_{\alpha, q}\right)$ and $\ell^{2}\left(\mathbb{Z}, \tilde{m}_{\alpha}\right)$. Their spectra are both known to be the interval $[-\sqrt{4 \alpha(1-\alpha)}, \sqrt{4 \alpha(1-\alpha)}]$. The respective Plancherel measures are continuous with respect to Lebesgue measure on that interval; they are

$$
\begin{aligned}
& \frac{q+1}{2 \pi} \frac{\sqrt{4 \alpha(1-\alpha)-\lambda^{2}}}{\tau_{\alpha}^{2}-\lambda^{2}} d \lambda \text { with } \tau_{\alpha}=\frac{\sqrt{4 \alpha(1-\alpha)}}{2 \sqrt{q} /(q+1)} \text { for } P_{\alpha, q} \text { on } \mathbb{T}_{q}, \\
& \frac{1}{\pi} \frac{1}{\sqrt{4 \alpha(1-\alpha)-\lambda^{2}}} d \lambda \text { for } \widetilde{P}_{\alpha} \text { on } \mathbb{Z} .
\end{aligned}
$$

This follows from the well known results for SRW on $\mathbb{T}_{q}$ and $\mathbb{Z}$ (see e.g., [28], p. 192 and (19.27) on p. 214). To adapt the latter methods to the $\alpha$-walks, one can use the same method as used above for Corollary 4, conjugating as in (7.3) with the square root of the reversing measure. The corresponding dilation factors are $\tau_{\alpha}$ when passing to $P_{\alpha, q}$ from SRW on $\mathbb{T}_{q}$ (whose spectral radius is the denominator of $\tau_{\alpha}$ above), and $\sqrt{4 \alpha(1-\alpha)}$ when passing to $\widetilde{P}_{\alpha}$ from SRW on $\mathbb{Z}$.

The interesting fact to observe here is that the projections preserve the spectrum, while the respective Plancherel measures differ drastically, starting with a sum of point masses over a dense, countable subset, and ending up with measures having continuous densities with respect to Lebesgue measure.

C. Green kernel estimates. Using the spectral measures, one may also undertake a computation of the asymptotic behavior of the Green kernel

$$
G_{\alpha}(o, x)=\sum_{N=0}^{\infty} p^{N}(x, y)=\int_{\operatorname{spec}\left(P_{\alpha}\right)} \frac{1}{1-\lambda} d \mu_{o, x}(\lambda), \quad x, y \in D L(q, r) .
$$

in space, i.e., as $d(o, x) \rightarrow \infty$. Due to the many oscillating terms that occur in these integrals, the spectral method becomes quite tedious, while a more probabilistic reasoning is more efficient and likely to admit extensions to more general random walks, see Brofferio and Woess [4]. The asymptotics depend on the way (direction) in which $x$ tends to $\infty$ in $D L$, and they also differ according to whether the drift is non-zero $(\alpha \neq 1 / 2)$ or zero $(\alpha=1 / 2)$. 


\section{References}

[1] Bartholdi, L. and Grigorchuk, R. I. (2000). On the spectrum of Hecke type operators related to some fractal groups, Proc. Steklov Inst. Math. 231, 1-41.

[2] Bartholdi, L., Grigorchuk, R. I., and Nekrashevych, V. (2003). From fractal groups to fractal sets, in Fractals in Graz 2001, Grabner, P. and Woess, W., Eds., 25-118, Birkhäuser, Basel.

[3] Bertacchi, D. (2001). Random walks on Diestel-Leader graphs, Abh. Math. Sem. Univ. Hamburg 71, $205-$ 224.

[4] Brofferio, S. and Woess, W. Green kernel estimates and the full Martin boundary for random walks on lamplighter groups and Diestel-Leader graphs, Ann. Inst. H. Poincaré Probab. Statist., to appear.

[5] Cartier, P. (1973). Harmonic analysis on trees, Proc. Sympos. Pure Math. 26, 419-424.

[6] Cartwright, D. I. (2001). Spherical harmonic analysis on buildings of type $\tilde{A}_{n}$, Monatsh. Math. 133, 93109.

[7] Cartwright, D. I. and Młotkowski, W. (1994). Harmonic analysis for groups acting on triangle buildings, J. Austral. Math. Soc. Ser. A 56, 345-383.

[8] Dicks, W. and Schick, Th. (2002). The spectral measure of certain elements of the complex group ring of a wreath product, Geom. Dedicata 93, 121-137.

[9] Diestel, R. and Leader, I. (2001). A conjecture concerning a limit of non-Cayley graphs, J. Algebraic Combin. 14, 17-25.

[10] Dodziuk, J. (1984). Difference equations, isoperimetric inequality, and transience of certain random walks, Trans. Amer. Math. Soc. 284, 787-794.

[11] Faraut, J. and Picardello, M. A. (1984). The Plancherel measure for symmetric graphs, Ann. Mat. Pura Appl. 138, 151-155.

[12] de la Harpe, P., Robertson, A. G., and Valette, A. (1993). On the spectrum of the sum of generators for a finitely generated group, Israel J. Math. 81, 65-96.

[13] Grigorchuk, R. I. and Żuk, A. (1999). On the asymptotic spectrum of random walks on infinite families of graphs, Sympos. Math. 39, 188-204.

[14] Grigorchuk, R. I. and Żuk, A. (2001). The lamplighter group as a group generated by a 2-state automaton, and its spectrum, Geom. Dedicata 87, 209-244.

[15] Kesten, H. (1959). Symmetric random walks on groups, Trans. Amer. Math. Soc. 92, 336-354.

[16] Krön, B. (2002). Green functions on self-similar graphs and bounds for the spectrum of the Laplacian, Ann. Inst. Fourier (Grenoble) 52, 1875-1900.

[17] Kuhn, G. and Soardi, P. M. (1983). The Plancherel measure for polygonal graphs, Ann. Mat. Pura Appl. 134, 393-401.

[18] Malozemov, L. and Teplyaev, A. (1995). Pure point spectrum of the Laplacians on fractal graphs, J. Funct. Anal. 129, 390-405.

[19] McKay, B. (1981). The expected eigenvalue distribution of a large regular graph, Linear Algebra Appl. 40, 203-216.

[20] Mohar, B. and Woess, W. (1989). A survey on spectra of infinite graphs, Bull. London Math. Soc. 21, 209-234.

[21] Pittet, C. and Saloff-Coste, L. (2002). On random walks on wreath products, Ann. Probab. 30, 948-977.

[22] Pólya, G. (1921). Über eine Aufgabe der Wahrscheinlichkeitsrechnung betreffend die Irrfahrt im Straßennetz, Mat. Ann. 84, 149-160.

[23] Revelle, D. (2003). Heat kernel asymptotics on the lamplighter group, Electron. Comm. Probab. 8, 142-154.

[24] Sabot, Ch. (2000). Pure point spectrum for the Laplacian on unbounded nested fractals, J. Funct. Anal. 173, 497-524.

[25] Saloff-Coste, L. and Woess, W. (1997). Transition operators, groups, norms, and spectral radii, Pacific J. Math. 180, 333-367.

[26] Serre, J.-P. (1997). Répartition asymptotique des valeurs propres de l'opérateur de Hecke $T_{p}$, J. Amer. Math. Soc. 10, 75-102.

[27] Teplyaev, A. (1998). Spectral analysis on infinite Sierpiński gaskets, J. Funct. Anal. 159, 537-567. 
[28] Woess, W. (2000). Random Walks on Infinite Graphs and Groups, Cambridge Tracts in Math. 138, Cambridge University Press, Cambridge.

[29] Woess, W. Lamplighters Diestel-Leader graphs, random walks, and harmonic functions, Combin. Probab. Comput., to appear.

Received November 12, 2003

IGAT, Bâtiment BCH, École Polytechnique Fédérale, CH-1015 Lausanne, Switzerland e-mail: laurent.bartholdi@epfl.ch

Institut für Mathematik C, Technische Universität Graz, Steyrergasse 30, A-8010 Graz, Austria

e-mail: woess@TUGraz.at 